\title{
A ORDEM DO SUBGRUPO UNITÁRIO DE ALGUMAS ÁLGEBRAS \\ DE GRUPO MODULARES
}

\author{
Antonio Luiz Rosa \\ TESE APRESENTADA \\ $\mathrm{AO}$ \\ INSTITUTO DE MATEMÁTICA E ESTATÍSTICA \\ DA \\ UNIVERSIDADE DE SÃO PAULO \\ PARA \\ OBTENÇÃO DO GRAU \\ $\mathrm{DE}$ \\ DOUTOR EM MATEMÁTICA \\ Área de Concentração: Álgebra \\ Orientador: Prof. Dr. Francisco César Polcino Milies
}

- São Paulo, outubro de 2000 - 


\section{A ORDEM DO SUBGRUPO UNITÁRIO \\ DE ALGUMAS ÁLGEBRAS DE GRUPO MODULARES}

Antonio Luiz Rosa

Este exemplar corresponde à redação final da tese apresentada por Antonio Luiz Rosa, devidamente corrigida e aprovada pela Comissão Julgadora

São Paulo, outubro de 2000

\section{Comissão Julgadora}

- Prof. Dr. Francisco César Polcino Milies - IME-USP

- Prof. Dr. Jairo Zacarias Gonçalves - IME-USP

- Prof. Dr. Guilherme Leal - UFRJ

- Prof. Dr. Rudolf Meier - UnB

- Prof. Dr. Miguel Ferrero - UFRGS 
"Ainda que eu falasse as línguas dos homens e dos anjos, se não tiver amor,

sou como o bronze que soa, ou como o címbalo que retine.

Mesmo que eu tivesse o dom da profecia, e conhecesse todos os mistérios e toda a ciência; mesmo que eu tivesse toda a fé, a ponto de transportar montanhas, se não tiver amor, não sou nada." 
À minha

maravilhosa

família. 


\section{Agradecimentos}

A Deus que me concedeu esta oportunidade, privilégio e alegria;

À minha família pelo apoio durante a realização deste;

Aos professores do IME-USP que muito contribuíram para a minha formação;

Aos professores e amigos da Universidade Federal de Ouro Preto que muito me encorajaram no êxito deste trabalho;

E a todos os demais que de forma direta ou indireta contribuíram para o êxito deste meu curso;

De forma especial, agradeço ao meu orientador, Prof. Dr. Francisco César Polcino Milies, pela confiança, pela paciência e sobretudo pelos ensinamentos no decorrer destes anos de saudável convívio;

E, ainda, em caráter especial, agradeço ao Prof. Dr. Victor Bovdi e ao Prof. Dr. Adalbert Bovdi, ambos da Lajos Kossuth University-Debrecen-Hungria, pela amizade, pela atenção, pelas informações e por tudo mais que foi fundamental para a realização deste trabalho. 


\section{RESUMO}

Sejam $K$ um corpo, $G$ um grupo e $V(K G)$ o grupo das unidades normalizadas na álgebra de grupo $K G$. O anti-automorfismo em $G, g \mapsto g^{-1}$, estende-se linearmente ao anti-automorfismo

$$
\alpha=\sum \alpha_{g} g \mapsto \alpha^{*}=\sum \alpha_{g} g^{-1}
$$

em $V(K G)$. Define-se o subgrupo unitário de $K G$ o seguinte:

$$
V_{*}(K G)=\left\{\alpha \in V(K G) \mid \alpha^{*}=\alpha^{-1}\right\}
$$

O objetivo deste trabalho é determinar a ordem do subgrupo unitário $V_{*}(K G)$, onde $K$ é um corpo finito de característica $p$ ( $p$ um número primo) e $G$ é um $p$-grupo finito.

Numa primeira etapa, determinamos a ordem do subgrupo unitário $V_{*}(K G)$ no caso da característica $p>2$. No restante deste trabalho, determinamos a ordem do subgrupo unitário $V_{*}(K G)$, quando $K$ é um corpo finito de característica 2 e $G$ é um grupo finito dentre os seguintes:

(i) G é um 2-grupo extra-especial;

(ii) G é um produto central de um 2-grupo extra-especial com um grupo cíclico de ordem 4

(iii) $G$ é um 2-grupo contendo um subgrupo abeliano $A$ de índice 2 e um elemento $b$ tal que $b$ inverte cada elemento de $A$.

Ressaltamos que por (iii) obtemos a ordem do subgrupo unitário $V_{*}(K G)$ para os 2-grupos diedrais $D_{2^{n}}$ e os quatérnios generalizados $Q_{2^{n}}(n \geq 3)$. 


\section{ABSTRACT}

Let $K$ be a field, $G$ be a group and $V(K G)$ be the group of normalized units in the group algebra $K G$. The anti-automorphism in $G, g \mapsto g^{-1}$, extends linearly to an anti-automorphism

$$
\alpha=\sum \alpha_{g} g \mapsto \alpha^{*}=\sum \alpha_{g} g^{-1}
$$

of $V(K G)$. Define the unitary subgroup of $K G$ the following

$$
V_{*}(K G)=\left\{\alpha \in V(K G) \mid \alpha^{*}=\alpha^{-1}\right\}
$$

We determine in this work the order of unitary subgroup $V_{*}(K G)$, where $K$ is a finite field of characteristic $p$ ( $p$ is a number prime) and $G$ is a finite $p$-group.

In a first step, we determine the order of unitary subgroup $V_{*}(K G)$ in the case of the characteristic $p>2$. In the remainder of this work, we determine the order of unitary subgroup $V_{*}(K G)$, when $K$ is a finite field of characteristic 2 and $G$ is a finite $p$-group among the following:

(i) $G$ is an extraspecial 2-group;

(ii) $G$ is a central product of an extraspecial 2-group with a cyclic group of order 4;

(iii) $G$ is a 2-group which contains an abelian subgroup $A$ of index two and an element $b$ such that $b^{-1} a b=a^{-1}$ for all $a \in A$.

We emphasize that by (iii), we obtain the order of unitary subgroups $V_{*}(K G)$ for all dihedral group $D_{2^{n}}$ and for all generalized quaternion $Q_{2^{n}}(n \geq 3)$. 


\section{Sumário}

Introdução

1 Resultados preliminares 4

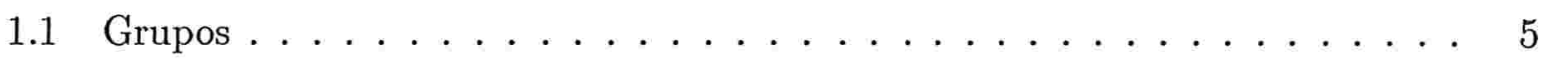

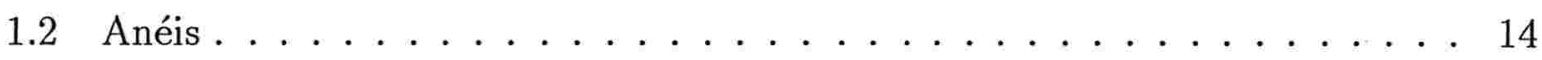

1.3 Anéis de Grupos . . . . . . . . . . . . . . . . . . 18

2 A ordem do subgrupo unitário para característica $p>2 \quad 25$

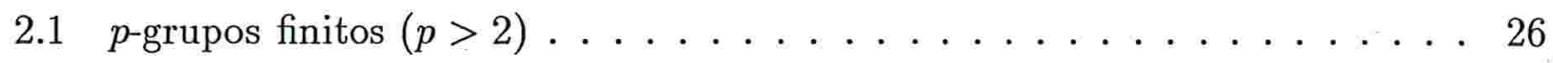

3 A ordem do subgrupo unitário para alguns produtos centrais $\quad 33$

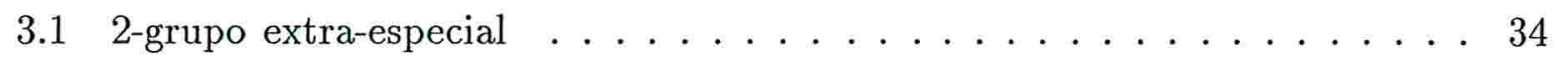

3.2 Produto central de um 2-grupo extra-especial com um grupo cíclico de

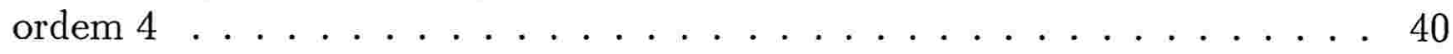

4 A ordem do subgrupo unitário para os 2-grupos diedrais e para os quatérnios generalizados $\quad 46$

4.1 2-grupos diedrais . . . . . . . . . . . . . . . . . 47

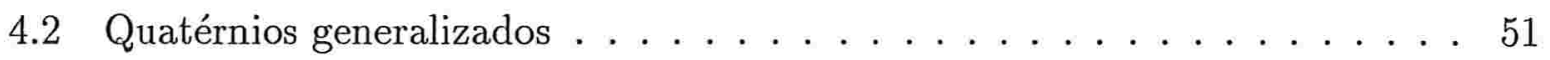

$\begin{array}{ll}\text { Referências } & \mathbf{5 7}\end{array}$ 


\section{Notação}

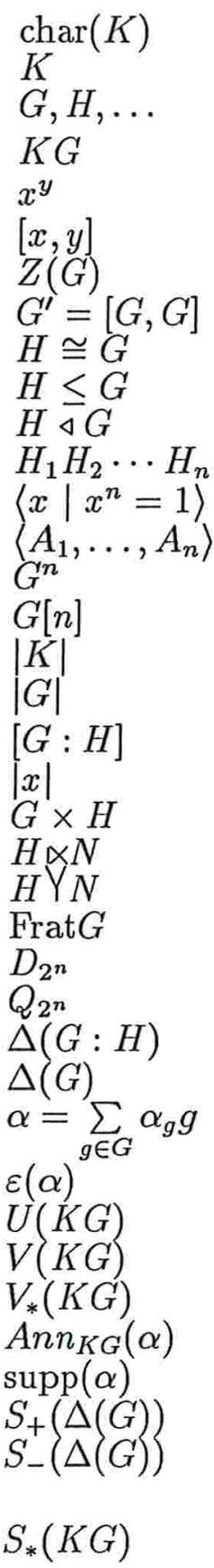

Característica do corpo $K$

Corpo

Grupos

Álgebra de grupo de um grupo $G$ sobre um corpo $K$

$y^{-1} x y$

$x^{-1} y^{-1} x y$

Centro do grupo $G$

Subgrupo comutador de um grupo $G$

$H$ é isomorfo $\operatorname{com} G$

$H$ é um subgrupo do grupo $G$

$H$ é um subgrupo normal de $G$

Produto de subconjuntos de um grupo

Subgrupo gerado pelo elemento $x$ de ordem $n$

Subgrupo gerado pelo conjunto $A_{1}, \ldots, A_{n}$

Subgrupo gerado por todos $g^{n}$, onde $g \in G$

Subgrupo gerado por todos $g \in G$ tal que $g^{n}=1$

Ordem do corpo $K$

Ordem do grupo $G$

Índice do subgrupo $H$ no grupo $G$

Ordem do elemento $x$

Produto direto

Produto semidireto

Produto central

Subgrupo de Frattini de $G$

Grupo diedral de ordem $2^{n}$

Grupo quatérnio generalizado de ordem $2^{n}$

Ideal de $K G$ gerado pelos elementos $h-1$, onde $h \in H$

Ideal de aumento de $K G$

Elemento na álgebra $K G$ onde $\alpha_{g} \in K$ para todo $g \in G$

Aumento do elemento $\alpha \in K G$

Grupo das unidades de $K G$

Grupo das unidades normalizadas de $K G$

Subgrupo unitário de $K G$

Anulador do elemento $\alpha$ em $K G$

Suporte do elemento $\alpha$ em $K G$

Conjunto de elementos simétricos de aumento zero em $K G$

Conjunto de elementos anti-simétricos de aumento zero em $K G$

Conjunto das unidades normalizadas simétricas em $K G$ 


\section{Introdução}

O Subgrupo Unitário $V_{*}(K G)$ tornou-se um objeto de grande interesse dentro da teoria de Álgebras de Grupo Modulares, no sentido de que o conhecimento deste aproxima-nos do conhecimento do grupo das unidades normalizadas $V(K G)$ e de possíveis respostas a muitos problemas abertos nesta teoria.

Em 1995, A. Bovdi e A. Szakács [4,5] descreveram plenamente a estrutura de $V_{*}(K G)$, dando uma base para este grupo, quando $K G$ é uma álgebra de grupo de um $p$-grupo abeliano finito $G$ sobre um corpo finito $K$ de $p^{m}$ elementos.

Muito pouco se sabe no caso em que $G$ é um p-grupo finito não abeliano (vide [1]). Pode-se considerar que um primeiro passo nesta direção é um resultado de V. Bovdi e L.G. Kovács [6]. Eles determinaram condições sobre a álgebra $\mathbb{F}_{p} G$, de um $p$-grupo não abeliano $G$, nas quais as unidades bicíclicas de $V\left(\mathbb{F}_{p} G\right)$ são unitárias.

Muitos problemas ainda permanecem abertos sobre $V_{*}(K G)$ quando $G$ é não abeliano. Podemos citar dentre muitos os seguintes: 
(i) Determinar um sistema de geradores para $V_{*}(K G)$.

(ii) Quando é que $G$ tem um complemento normal em $V_{*}(K G)$ ?

(iii) Qual a classe de nilpotência de $V_{*}(K G)$ ?

(iv) Se $K G$ é finito, qual é a ordem de $V_{*}(K G)$ ?

É claro que, para responder as questões (i), (ii) ou (iii) resulta necessário primeiro responder (iv).

Neste trabalho, vamos determinar a ordem de $V_{*}(K G)$ quando a característica de $K$ é $p>2, p$ primo, para todas as famílias de $p$-grupos finitos não abelianos. Já no caso mais complexo da característica $p=2$, determinamos a ordem de $V_{*}(K G)$ para diversas famílias de 2-grupos finitos não abelianos.

A tese é apresentada em quatro capítulos. O primeiro inclui noções preliminares que serão usadas em resultados dos capítulos seguintes. Este se divide em resultados preliminares sobre a Teoria dos Grupos, a Teoria de Anéis e em resultados preliminares sobre a Teoria de Anéis de Grupo. Nosso propósito aqui não é uma completa dissertação sobre tais assuntos, mas simplesmente recordarmos o essencial para uma completa compreensão dos resultados obtidos nos capítulos seguintes.

No segundo capítulo, determinamos a ordem do subgrupo unitário $V_{*}(K G)$ no caso em que a característica de $K$ é $p>2$. Aqui, não há restrição a nenhum $p$-grupo finito 
não abeliano $G$.

No terceiro capítulo, determinamos a ordem do subgrupo unitário $V_{*}(K G)$ quando $G$ é um determinado produto central. Incluímos aqui o caso em que $G$ é um 2-grupo extra-especial.

Por fim, no quarto capítulo, determinamos a ordem de $V_{*}(K G)$ quando $G$ contém um subgrupo abeliano $A$ de índice dois e um elemento $b$ que inverte cada elemento de $A$. Como casos particulares, determinamos a ordem de $V_{*}(K G)$ quando $G$ é do tipo 2-grupo diedral ou um quatérnio generalizado.

Os resultados aqui contidos foram recentemente publicados no Communications in Algebra [9]. 


\section{Capítulo 1}

\section{Resultados preliminares}

Neste capítulo apresentamos resultados básicos que serão utilizados nos capítulos seguintes.

Começamos expondo a parte sobre grupos e anéis, recordando apenas o que for essencial para a compreensão dos mesmos. Em seguida, expomos a parte referente a Anéis de Grupos, em que recordamos os principais resultados obtidos até o momento e que serão fundamentais em nossas conclusões. 


\subsection{Grupos}

Seja $G$ um grupo. O subgrupo de Frattini de $G$ é, por definição, a intersecção de todos os seus subgrupos maximais. Denotaremos este subgrupo por Frat $G$.

Teorema 1.1.1 (Wielandt) Seja $G$ um grupo finito. Então $G$ é nilpotente se e somente se $G^{\prime} \leq \operatorname{Frat} G$.

Prova: [pp.137, 11].

Teorema 1.1.2 (Burnside) Seja $G$ um p-grupo finito. Então Frat $G=G^{\prime} G^{p}$.

Prova: [pp.140, 11].

Um p-grupo finito $G$ é dito ser um grupo extra-especial se $G^{\prime}$ e $Z(G)$, o subgrupo comutador e o centro de $G$, respectivamente, coincidem e têm ordem $p$.

Lema 1.1.3 Seja $G$ um grupo e u,v elementos de $G$. Então vale a identidade

$$
\left[u^{m}, v\right]=[u, v]^{u^{m-1}+u^{m-2}+\cdots+u^{2}+u+1} \quad(m \in \mathbb{N}) .
$$

Além disso, se $[u, v]$ pertence ao centro de $\langle u, v\rangle$, então $\left[u^{m}, v\right]=[u, v]^{m}=\left[u, v^{m}\right]$. 
Prova: Se $m=1$, é evidente a identidade. Agora temos que

$$
\left[u^{m}, v\right]=\left[u^{m-1} \cdot u, v\right]=\left[u^{m-1}, v\right]^{u} \cdot[u, v]
$$

e o resultado segue por indução.

Teorema 1.1.4 Seja $G$ um p-grupo extraespecial. Então Frat $G, Z(G)$ e $G^{\prime}$ coincidem e têm ordem $p$.

Prova: Que $Z(G)$ e $G^{\prime}$ coincidem e têm ordem $p$ segue por definição. Agora, pelo Teorema 1.1.2, temos que Frat $G=G^{\prime} G^{p}$, e pelo Lema 1.1.3, segue que para todos $x, g \in G$, $\left[x, g^{p}\right]=[x, g]^{p}=1$ e disto $g^{p} \in Z(G)=G^{\prime}$. Portanto, $G^{p}=\left\langle g^{p} \mid g \in G\right\rangle \subseteq G^{\prime}$ e disto Frat $G=G^{\prime}$.

Um grupo $G$ é dito ser um produto central de seus subgrupos normais $G_{1}, \ldots, G_{n}$ se:

1. $G=G_{1} \cdots G_{n}$

2. $\left[G_{i}, G_{j}\right]=1$, para $i \neq j$;

3. $G_{i} \cap \prod_{j \neq i} G_{j}=C \subseteq Z(G)$, para todo $i$.

Ao subgrupo $C$, chamaremos de subgrupo fator central. 
Como $C \subseteq Z(G)$ então $C \subseteq Z\left(G_{i}\right)$, para todo $i$.

Notaremos $G=G_{1} Y_{G_{2}} \bigvee \ldots Y_{G_{n}}$.

Temos,

$$
|G|=\left(\prod_{i=1}^{n}\left|G_{i}\right|\right) /|C|
$$

Temos ainda que,

$$
\bigcup_{i=1}^{n} Z\left(G_{i}\right)=Z(G)
$$

Observamos que todo produto direto de grupos $G=H \times K$ pode ser visto como um produto central destes grupos $\operatorname{com} C=\langle 1\rangle$.

Exemplo 1: Sejam

$$
A=\left\langle a, b \mid a^{4}=1, a^{2}=b^{2}, a^{b}=a^{-1}\right\rangle=\left\{1, a, a^{2}, a^{3}, b, a b, a^{2} b, a^{3} b\right\}
$$

e

$$
\begin{gathered}
B=\left\langle c, d \mid c^{4}=1, d^{4}=1, d c=c^{3} d\right\rangle \\
B=\left\{1, c, c^{2}, c^{3}, d, c d, c^{2} d, c^{3} d, d^{2}, c d^{2}, c^{2} d^{2}, c^{3} d^{2}, d^{3}, c d^{3}, c^{2} d^{3}, c^{3} d^{3}\right\}
\end{gathered}
$$

Temos:

(i) $A=Q_{8},|A|=8, Z(A)=\left\{1, a^{2}\right\},|Z(A)|=2$; 
(ii) $B=\langle c\rangle \rtimes\langle d\rangle,\langle c\rangle \triangleleft B,|B|=16, Z(B)=\left\{1, c^{2}, d^{2}, c^{2} d^{2}\right\},|Z(B)|=4$.

Logo é possível obter 3 grupos do tipo $G=A Y_{B}$, a saber:

1. $G_{1}=A Y_{B}$ com $C=\left\langle a^{2} \mid a^{2}=c^{2}\right\rangle$, isto é,

$G_{1}=\langle a, b, c, d| a^{4}=c^{4}=d^{4}=1, a^{2}=b^{2}=c^{2}, d c=c^{3} d,[a, c]=[a, d]=[b, c]=$ $[b, d]=1\rangle$

2. $G_{2}=A Y_{B} \operatorname{com} C=\left\langle a^{2} \mid a^{2}=d^{2}\right\rangle$, isto é,

$G_{2}=\langle a, b, c, d| a^{4}=c^{4}=d^{4}=1, a^{2}=b^{2}=d^{2}, d c=c^{3} d,[a, c]=[a, d]=[b, c]=$ $[b, d]=1\rangle$;

3. $G_{3}=A Y_{B} \operatorname{com} C=\left\langle a^{2} \mid a^{2}=c^{2} d^{2}\right\rangle$, isto é,

$G_{1}=\langle a, b, c, d| a^{4}=c^{4}=d^{4}=1, a^{2}=b^{2}=c^{2} d^{2}, d c=c^{3} d,[a, c]=[a, d]=[b, c]=$ $[b, d]=1\rangle ;$

Apesar de $\left|G_{i}\right|=(8 \times 16) / 2=64$ para $i=1,2,3$, temos que $G_{1}, G_{2}$ e $G_{3}$ não são isomorfos.

Exemplo 2: Sejam

$$
A=H_{1} Y_{H_{2}} Y \ldots Y H_{n}, \quad H_{i}=\left\langle a_{i}, b_{i} \mid a_{i}^{4}=1, b_{i}{ }^{2}=a_{i}{ }^{2}, a_{i}{ }^{b_{i}}=a_{i}{ }^{-1}\right\rangle \cong Q_{8},
$$


um 2-grupo extra-especial, e

$$
B=\left\langle x \mid x^{4}=1\right\rangle, \text { um grupo cíclico de ordem } 4
$$

Temos:

(i) $|A|=2^{2 n+1}, Z(A)=\left\{1, a^{2}\right\},|Z(A)|=2$;

(ii) $|B|=4, Z(B)=B=\left\{1, x, x^{2}, x^{3}\right\},|Z(B)|=4$.

Logo é possível obter o seguinte grupo do tipo $G=A Y_{B}$ com $C=\left\langle a^{2} \mid a^{2}=x^{2}\right\rangle$, $|C|=2, C \subseteq Z(A)$ e $C \subseteq Z(B)$, a saber:

$$
G=\left\langle a_{i}, b_{i}, x \mid a_{i}^{4}=x^{4}=1, a_{i}^{2}=b_{i}^{2}=x^{2}, a_{i}^{b_{i}}=a_{i}^{-1},\left[a_{i}, x\right]=\left[b_{i}, x\right]=1\right\rangle .
$$

Temos ainda que

$$
|G|=\frac{2^{2 n+1} \times 4}{2}=2^{2 n+2}
$$

Lema 1.1.5 Se G é um grupo não abeliano de ordem $p^{3}$, então $G$ é extra-especial.

Prova: Sendo $G$ não abeliano, então $G \neq Z(G)$ e $G^{\prime} \neq 1$. Por outro lado, sendo $|G|=p^{3}$, então $Z(G) \neq 1$ e disto $|Z(G)|=p$ ou $p^{2}$. Mas se $|Z(G)|=p^{2}, G / Z(G)$ é cíclico e portanto $G$ abeliano, o que é uma contradição. Portanto $|Z(G)|=p$. 
Agora, como $G$ é um grupo nilpotente e $1 \neq G^{\prime} \triangleleft G$, então $G^{\prime} \cap Z(G) \neq 1$. Mas $G^{\prime} \cap Z(G) \subseteq Z(G)$ e disto $G^{\prime} \cap Z(G)=Z(G)$, i.e, $Z(G) \subseteq G^{\prime}$.

Por outro lado, $\left|\frac{G}{Z(G)}\right|=p^{2}$, i.e, $\frac{G}{Z(G)}$ é abeliano. Logo, $G^{\prime} \subseteq Z(G)$. Portanto,
$G^{\prime}=Z(G)$ e $\left|G^{\prime}\right|=|Z(G)|=p$.

É natural pensar um $p$-grupo extra-especial $G$ como um produto direto de grupos $G_{i}$ nos quais subgrupos centrais $\operatorname{dos} G_{i}$ são identificados por um subgrupo fator central $C$.

Teorema 1.1.6 Um p-grupo extra-especial é um produto central de $n$ subgrupos não abelianos de ordem $p^{3}$ e tem ordem $p^{2 n+1}$. Reciprocamente, um produto central finito de grupos não abelianos de ordem $p^{3}$ com subgrupo fator central não trivial é um p-grupo extra-especial.

Prova: [pp.146, 11].

Lema 1.1.7 Sejam $D_{8}$ o grupo diedral de ordem 8 e $Q_{8}$ o grupo quatérnio de ordem 8. Então

$$
D_{8} Y_{D_{8}} \cong Q_{8} Y_{Q_{8}}
$$

Prova: [pp.139, 12] Seja $D_{8}=\left\langle x, y \mid x^{4}=1=y^{2}, x^{y}=x^{-1}\right\rangle$. Deste modo, $D_{8}=$ $\left\{x^{i} y^{j} ; 0 \leq i \leq 3, j=0,1\right\}$ e temos a regra $\left(x^{i} y^{j}\right)\left(x^{k} y^{l}\right)=x^{a} y^{b}$, onde $a \equiv i+(-1)^{j} k$ 
$(\bmod 4)$ e $b \equiv j+l(\bmod 2)\left(\right.$ isto segue da relação $\left.x y=y x^{-1}\right)$.

Seja $x^{i} y^{j} \in Z\left(D_{8}\right), 0 \leq i \leq 3, j=0,1$. Então, visto que $D_{8}=\langle x, y\rangle$, devemos ter:

$$
\left(x^{i} y^{j}\right) x=x\left(x^{i} y^{j}\right) \Rightarrow x^{i+(-1)^{j}} \cdot y^{j}=x^{1+i} y^{j} \Rightarrow x^{(-1)^{j}-1}=1
$$

e

$$
\left(x^{i} y^{j}\right) y=y\left(x^{i} y^{j}\right) \Rightarrow x^{i} y^{1+j}=x^{-i} y^{1+j} \Rightarrow x^{2 i}=1
$$

Logo, devemos ter $(-1)^{j} \equiv 1(\bmod 4)$ e $2 i \equiv 0(\bmod 4)$, com $0 \leq i \leq 3$ e $j=0,1$. Disto segue que $j=0, i=2$ e $x^{i} y^{j} \in Z\left(D_{8}\right)$ com $x^{i} y^{j}=x^{2}$. Portanto, temos $Z\left(D_{8}\right)=\left\langle x^{2}\right\rangle$.

Sejam $D_{i}(i=1,2)$ grupos isomorfos a $D_{8}$. Consideramos o produto central $G=$ $D_{1} Y_{D_{2}}$ identificando o centro. Isto é, $\left[D_{1}, D_{2}\right]=1, G=D_{1} D_{2}$ e $Z\left(D_{8}\right)=D_{1} \cap D_{2}$.

Sejam $D_{1}=\left\langle x_{1}, y_{1}\right\rangle$ e $D_{2}=\left\langle x_{2}, y_{2}\right\rangle$. Identificando os centros, fazemos $x_{1}^{2}=x_{2}^{2}=c$.

Definimos agora dois subgrupos $Q_{1}$ e $Q_{2}$ por

$$
Q_{1}=\left\langle x_{1}, y_{1} x_{2}\right\rangle, \quad Q_{2}=\left\langle x_{1} y_{2}, x_{2}\right\rangle
$$

Faça $x_{3}=y_{1} x_{2}$ e $x_{4}=x_{1} y_{2}$. Visto que $\left[D_{1}, D_{2}\right]=1$, temos:

$$
\begin{aligned}
x_{3}^{2} & =\left(y_{1} x_{2}\right)\left(y_{1} x_{2}\right)=y_{1}^{2} x_{2}^{2}=x_{2}^{2}=c \\
x_{4}^{2} & =\left(x_{1} y_{2}\right)\left(x_{1} y_{2}\right)=x_{1}^{2} y_{2}^{2}=x_{1}^{2}=c \\
x_{3}^{-1} x_{1} x_{3} & =\left(y_{1} x_{2}\right)^{-1} x_{1}\left(y_{1} x_{2}\right)=x_{2}^{-1} y_{1}^{-1} x_{1} y_{1} x_{2}
\end{aligned}
$$




$$
\begin{aligned}
& =x_{2}^{-1} x_{1}^{-1} x_{2}=x_{1}^{-1} \\
x_{4}^{-1} x_{2} x_{4} & =\left(x_{1} y_{2}\right)^{-1} x_{2}\left(x_{1} y_{2}\right)=y_{2}^{-1} x_{1}^{-1} x_{2} x_{1} y_{2} \\
& =y_{2}^{-1} x_{2} y_{2}=x_{2}^{-1} \\
\left(x_{1} x_{3}\right)^{2} & =\left(x_{1} x_{3}\right)\left(x_{1} x_{3}\right)=\left(x_{1} y_{1} x_{2}\right)\left(x_{1} y_{1} x_{2}\right) \\
& =y_{1} x_{1}^{-1} x_{2} x_{1} y_{1} x_{2}=y_{1} x_{2} y_{1} x_{2}=x_{3}^{2}=c \\
\left(x_{2} x_{4}\right)^{2} & =\left(x_{2} x_{4}\right)\left(x_{2} x_{4}\right)=\left(x_{2} x_{1} y_{2}\right)\left(x_{2} x_{1} y_{2}\right) \\
& =x_{2} x_{1} x_{2}^{-1} y_{2} x_{1} y_{2}=x_{1} y_{2} x_{1} y_{2}=x_{4}^{2}=c .
\end{aligned}
$$

Logo,

$$
Q_{1}=\left\langle x_{1}, x_{3} \mid x_{1}^{4}=1, x_{3}^{2}=x_{1}^{2}, x_{1}^{x_{3}}=x_{1}^{-1}\right\rangle \cong Q_{8}
$$

e

$$
Q_{2}=\left\langle x_{2}, x_{4} \mid x_{2}^{4}=1, x_{4}^{2}=x_{2}^{2}, x_{2}^{x_{4}}=x_{2}^{-1}\right\rangle \cong Q_{8}
$$

Portanto, temos $\left|Q_{1}\right|=\left|Q_{2}\right|=8$ e o único elemento de ordem 2 em $Q_{i}, i=1,2$, é $c$. Além do mais, valem:

$$
\begin{aligned}
x_{1} \cdot x_{4} & =x_{1} \cdot x_{1} y_{2}=x_{1} y_{2} x_{1}=x_{4} \cdot x_{1} \\
x_{2} x_{3} & =x_{2} y_{1} x_{2}=y_{1} x_{2} x_{2}=x_{3} x_{2} \\
x_{3} x_{4} & =\left(y_{1} x_{2}\right)\left(x_{1} y_{2}\right)=y_{1} x_{1} x_{2} y_{2}=x_{1}^{-1} y_{1} y_{2} x_{2}^{-1} \\
& =x_{1} x_{1}^{2} y_{1} y_{2} x_{2} x_{2}^{2}=x_{1} c y_{1} y_{2} x_{2} c=x_{1} y_{1} y_{2} x_{2} c^{2} \\
& =x_{1} y_{1} y_{2} x_{2}=x_{1} y_{2} y_{1} x_{2}=x_{4} x_{3} .
\end{aligned}
$$

Assim também $G=Q_{1} Y Q_{2}$. 
Como nem $D_{8}$ e nem $Q_{i}$ podem ser escritos como um produto central de subgrupos próprios, temos que $D_{8} Y_{D_{8}} \cong Q_{8} Y Q_{8}$.

Corolário 1.1.8 Seja $G$ um grupo extra-especial de ordem $2^{2 n+1}$. Então $G$ é um produto central de grupos, todos eles isomorfos a $D_{8}$, ou um produto central de cópias de $D_{8}$ com $u m Q_{8}$.

Assim, se $G$ é um grupo extra-especial de ordem $2^{2 n+1}$, então $G$ pode ser visto como

$$
G=G_{1} Y \ldots Y_{G_{n}}, \quad \operatorname{com} G_{i} \cong Q_{8}, \quad 1 \leq i \leq n .
$$

Agora, se $G_{i}=\left\langle a_{i}, b_{i}\right\rangle$ e $Z(G)=G^{\prime}=\left\langle c \mid c^{2}=1\right\rangle$, então cada elemento em $G$ pode ser escrito como

$$
x=c^{k} a_{1}^{\alpha_{1}} b_{1}^{\beta_{1}} a_{2}^{\alpha_{2}} b_{2}^{\beta_{2}} \cdots a_{n}^{\alpha_{n}} b_{n}^{\beta_{n}},
$$

$\operatorname{com} k=0,1, \alpha_{i}=0,1$ e $\beta_{i}=0,1,1 \leq i \leq n$, visto que $a_{i}^{-1}=a_{i}^{3}=c a_{i}$ e $b_{i}^{-1}=b_{i}^{3}=c b_{i}$, $1 \leq i \leq n$. Então, temos $x^{2}=b_{1}^{2 \beta_{1}} \cdots b_{n}^{2 \beta_{n}}=c^{\lambda}$, com $\lambda=0,1$, pois $a_{i}^{b_{i}}=a_{i}^{-1} \mathrm{e}$ $g^{2} \in Z(G)=G^{\prime}$, para todo $g \in G_{i}, i$ qualquer. Assim, a ordem de $x$ é 4 se $\lambda=1$ e $\lambda$ será 0 se a ordem de $x$ é 2 . De qualquer forma, cada elemento de $G$ pode ser escrito

$$
x=z_{i_{1}} z_{i_{2}} \cdots z_{i_{k}},
$$

onde $z_{i_{t}} \in G_{i_{t}}$ tem ordem 4 e $i_{1}<i_{2}<\cdots<i_{k}$. 


\subsection{Anéis}

Seja $R$ um anel com unidade 1 . Uma involução * sobre $R$ é uma aplicação $x \mapsto x^{*}$ que é um anti-automorfismo de período 2 . Isto é, * é um anti-automorfismo sobre $R$ tal que

$$
\left(r_{1} r_{2}\right)^{*}=r_{2}^{*} r_{1}^{*} \text { e }\left(r_{i}^{*}\right)^{*}=r_{i} \quad\left(r_{i} \in R\right)
$$

Um elemento $u \in R$ é chamado um elemento unitário se $u u^{*}=1=u^{*} u$. Um elemento $k \in R$ é chamado anti-simétrico se $k^{*}=-k$.

Lema 1.2.1 Seja $k$ um elemento anti-simétrico em $R$ tal que $1+k$ seja inversível em $R$. Então o elemento $u=(1-k)(1+k)^{-1}$ é unitário.

Prova: Primeiro, observamos que

$$
(1+k)(1-k)=1-k^{2}=(1-k)(1+k)
$$

Visto que $1+k$ é inversível, segue também que

$$
(1-k)(1+k)^{-1}=(1+k)^{-1}(1-k) .
$$

Assim, tomando

$$
u=(1-k)(1+k)^{-1}=(1+k)^{-1}(1-k) \in R,
$$

obtemos

$$
u(1+k)=1-k=(1+k) u
$$




$$
\begin{gathered}
u(1+k)=1-k \\
{[u(1+k)]^{*}=(1-k)^{*}} \\
(1+k)^{*} u^{*}=(1-k)^{*} \\
\left(1+k^{*}\right) u^{*}=\left(1-k^{*}\right) \\
(1-k) u^{*}=1+k .
\end{gathered}
$$

Além disso, usando (1) obtemos

$$
\begin{aligned}
u^{*}(1-k) & =u^{*}\left(1+k^{*}\right)=u^{*}(1+k)^{*}=[(1+k) u]^{*} \\
& =(1-k)^{*}=\left(1-k^{*}\right)=1+k=(1-k) u^{*}
\end{aligned}
$$

Portanto,

$$
\begin{aligned}
u u^{*} & =\left[(1+k)^{-1}(1-k)\right] u^{*}=(1+k)^{-1}\left[(1-k) u^{*}\right] \\
& =(1+k)^{-1}(1+k)=1
\end{aligned}
$$

$\mathrm{e}$

$$
\begin{aligned}
u^{*} u & =u^{*}\left[(1-k)(1+k)^{-1}\right]=\left[u^{*}(1-k)\right](1+k)^{-1} \\
& =(1+k)(1+k)^{-1}=1
\end{aligned}
$$

Os elementos unitários da forma $u=(1-k)(1+k)^{-1}$, onde $k$ é um elemento antisimétrico tal que $1+k$ é inversível em $R$, são chamados elementos unitários de Cayley. 
Lema 1.2.2 Seja $R$ um anel com involução * no qual 2 é inversível. Então um elemento unitário u é um elemento unitário de Cayley se, e somente se, $1+u$ é inversível em $R$.

Prova: [10] Suponha que $u$ seja um elemento unitário de Cayley. Isto é, $u=(1-k)(1+$ $k)^{-1}$ para algum elemento anti-simétrico $k$ tal que $1+k$ seja inversível em $R$.

Assim, temos

$$
\begin{gathered}
(1-k)=2-(1+k) \\
(1-k)(1+k)^{-1}=[2-(1+k)](1+k)^{-1} \\
u=2(1+k)^{-1}-1 \\
u+1=2(1+k)^{-1} .
\end{gathered}
$$

Logo, vê-se que $1+u$ é inversível em $R$.

Reciprocamente, suponha que $u$ seja um elemento unitário tal que $1+u$ seja inversível em $R$.

Considere $k=(1-u)(1+u)^{-1}$. Vemos então

$$
\begin{gathered}
k(1+u)=(1-u) \\
{[k(1+u)]^{*}=(1-u)^{*}} \\
(1+u)^{*} k^{*}=1-u^{*} \\
\left(1+u^{*}\right) k^{*}=1-u^{*}
\end{gathered}
$$




$$
\begin{gathered}
u\left[\left(1+u^{*}\right) k^{*}\right]=u\left(1-u^{*}\right) \\
{\left[u\left(1+u^{*}\right)\right] k^{*}=u-1} \\
(u+1) k^{*}=u-1 \\
k^{*}=-(1+u)^{-1}(1-u)=-(1-u)(1+u)^{-1}=-k,
\end{gathered}
$$

isto é, $k$ é um elemento anti-simétrico em $R$. Além do mais, $1+k=2(1+u)^{-1}$ é inversível em $R$.

Assim,

$$
\begin{gathered}
1+k=2(1+u)^{-1} \\
(1+k)(1+u)=2 \\
(1+k)+(1+k) u=2 \\
(1+k) u=2-(1+k) \\
(1+k) u=1-k \\
u=(1+k)^{-1}(1-k)=(1-k)(1+k)^{-1},
\end{gathered}
$$

isto é, $u$ é um elemento unitário de Cayley. 


\subsection{Anéis de Grupos}

Sejam $K$ um corpo e $G$ um grupo. A álgebra de grupo $K G$ de $G$ sobre $K$ é definida como sendo o $K$-módulo livre sobre os elementos de $G$, com a multiplicação induzida pela multiplicação de $G$, i.e, $K G$ consiste de todas as somas formais finitas da forma

$$
\alpha=\sum_{g \in G} \alpha_{g} g, \quad \alpha_{g} \in K,
$$

com a soma, multiplicação e multiplicação por escalar dadas por:

(i) $\sum_{g \in G} \alpha_{g} g+\sum_{g \in G} \beta_{g} g=\sum_{g \in G}\left(\alpha_{g}+\beta_{g}\right) g$;

(ii) $\left(\sum_{g \in G} \alpha_{g} g\right) \cdot\left(\sum_{h \in G} \beta_{h} h\right)=\sum_{g, h \in G} \alpha_{g} \beta_{h} g h=\sum_{t \in G} \delta_{t} t$,

onde $\delta_{t}=\sum_{g h=t} \alpha_{g} \beta_{h}$;

(iii) $\lambda\left(\sum_{g \in G} \alpha_{g} g\right)=\sum_{g \in G}\left(\lambda \alpha_{g}\right) g$, para todo $\lambda \in K$.

Para qualquer elemento $\alpha=\sum_{i=1}^{r} \alpha_{i} g_{i}$ em $K G$, define-se o suporte de $\alpha$ como sendo o conjunto $\operatorname{supp}(\alpha)=\left\{g_{i} \in G \mid \alpha_{i} \neq 0\right\}$.

Seja $H$ um subgrupo normal de $G$. Definimos $\Delta(G, H)$ como sendo o ideal de $K G$ gerado pelo conjunto $\{h-1 \mid h \in H\}$. Se $H=G$, então denotamos $\Delta(G, G)$ por $\Delta(G)$ e o chamamos de ideal de aumento de $K G$. Note que $\Delta(G)$ é o núcleo do homomorfismo

$$
\varepsilon: K G \rightarrow K
$$


definido por $\varepsilon\left(\sum_{g \in G} \alpha_{g} g\right)=\sum \alpha_{g}$. Este homomorfismo é chamado o homomorfismo aumento.

O conjunto de unidades em $K G$ forma um grupo denotado $U(K G)$, o grupo das unidades de $K G$. Se $\alpha \in U(K G)$, então $\varepsilon(\alpha) \in K^{*}=K \backslash\{0\}$. Definimos $V(K G)$ como sendo o conjunto

$$
\{\alpha \in U(K G) \mid \varepsilon(\alpha)=1\}
$$

que, obviamente, é um subgrupo de $U(K G) . V(K G)$ é chamado o grupo das unidades normalizadas em $K G$.

$\mathrm{O}$ anti-automorfismo em $G, g \mapsto g^{-1}$, estende-se linearmente a um anti-automorfismo $\alpha \mapsto \alpha^{*}$ de $K G$, onde se $\alpha=\sum_{g \in G} \alpha_{g} g$, então $\alpha^{*}=\sum_{g \in G} \alpha_{g} g^{-1}$. É fácil observar que $V(K G)$ é invariante sob este anti-automorfismo. Além disso, a restrição desta aplicação a $V(K G)$ seguido por $v \mapsto v^{-1}$ dá-nos um automorfismo de $V(K G)$. Os elementos de $V(K G)$ fixados por este automorfismo são as unidades normalizadas unitárias de $K G$, e estas formam um subgrupo ao qual denotamos por $V_{*}(K G)$, o subgrupo unitário de $K G$. Em outras palavras, temos:

$$
V_{*}(K G)=\left\{\alpha \in V(K G) \mid \alpha^{*}=\alpha^{-1}\right\}
$$

O subgrupo unitário $V_{*}(K G)$ será o objeto de nosso estudo neste trabalho.

Consideramos o caso modular em que $K$ é um corpo finito de característica prima $p$ e $G$ é um p-grupo finito. É bem sabido que nestas condições temos: 
(i) $|K G|=|K|^{|G|}$;

(ii) $\Delta(G)=\{\alpha \in K G \mid \varepsilon(\alpha)=0\}$ é um ideal nilpotente;

(iii) $U(K G)=\{\alpha \in K G \mid \varepsilon(\alpha) \neq 0\}$;

(iv) $K G=U(K G) \cup \Delta(G)$;

(v) $V(K G)=1+\Delta(G)$

(vi) $|V(K G)|=|K|^{|G|-1}$.

A estrutura do grupo $V_{*}(K G)$ de uma álgebra de grupo de um $p$-grupo abeliano finito $G$ sobre um corpo finito $K$ de $p^{m}$ elementos foi plenamente determinada por A. Bovdi e A. Szakács em [4,5]. Já sobre $V_{*}(K G)$ quando $G$ é não abeliano pouco se sabe (vide [1]). A. Bovdi e L. Erdei [2] descreveram o subgrupo unitário $V_{*}\left(\mathbb{F}_{2} G\right)$ para todos os grupos de ordem 8 e 16 .

Um dos primeiros resultados na investigação do subgrupo unitário $V_{*}(K G)$ no caso não abeliano pode ser considerado o seguinte resultado devido a V. Bovdi e T. Rozgonyi $[8]$.

Teorema 1.3.1 Seja $G$ um 2-grupo finito contendo um subgrupo abeliano $A$ de índice 2. Suponha que exista um elemento $b \in G \backslash A$ de ordem 4 tal que $b^{-1} a b=a^{-1}$ para todo $a \in A$. Então o subgrupo unitário $V_{*}\left(\mathbb{F}_{2} G\right)$ é um produto semidireto de $G$ e um subgrupo 
normal H. O subgrupo $H$ é o produto semidireto do 2-grupo abeliano elementar normal

$$
W=\left\{1+\left(1+b^{2}\right) z b \mid z \in \mathbb{F}_{2} A\right\}
$$

e o subgrupo abeliano $L$, onde $V_{*}\left(\mathbb{F}_{2} A\right)=A \times L$. O grupo abeliano $W$ é o produto direto de $\frac{1}{2}|A|$ cópias do grupo aditivo do corpo $\mathbb{F}_{2}$.

Tal resultado foi baseado no seguinte importante resultado de A. Bovdi e A.A. Szakács [4].

Teorema 1.3.2 Sejam $K$ um corpo de $2^{m}$ elementos e $G$ um 2-grupo abeliano finito. Então:

(i) $V_{*}(K G)=G \times L$, onde $L$ é um subgrupo abeliano;

(ii) $\left|V_{*}(K G)\right|=\left|G^{2}[2]\right||K|^{\left.\frac{1}{2}(|G|+\mid G[2]]\right)-1}$, onde $G[2]=\left\{g \in G \mid g^{2}=1\right\}$.

Em seguida, listamos alguns resultados preliminares essenciais na obtenção de nossos novos resultados. Estes são devidos a V. Bovdi e L.G. Kovács [6].

Lema 1.3.3 Sejam $K$ um anel comutativo com 1 e $G$ um grupo qualquer. Para $x \in$ $V(K G)$ e $y \in V_{*}(K G)$, temos que $x^{-1} y x \in V_{*}(K G)$ se e somente se $x x^{*}$ comuta com $y$. 
Prova: Basta notarmos que $\left(x^{-1} y x\right)^{*}=\left(x^{-1} y x\right)^{-1}$ significa que $x^{*} y^{*}\left(x^{*}\right)^{-1}=x^{-1} y^{-1} x$, o que é equivalente a $x x^{*} y^{*}=y^{-1} x x^{*}$. Visto que por hipótese $y^{*}=y^{-1}$, segue o resultado.

É fácil observarmos que, visto que $G \leq V_{*}(K G)$, qualquer elemento $\alpha \in K G$ que comuta com cada elemento de $V_{*}(K G)$ é central em $K G$. Deste modo, o Lema 1.2.3 nos dá o seguinte:

Corolário 1.3.4 O subgrupo $V_{*}(K G)$ é normal em $V(K G)$ se e somente se todos os elementos da forma $x x^{*}$ com $x \in V(K G)$ são centrais em $K G$.

Teorema 1.3.5 Sejam K um corpo de característica p e G um p-grupo localmente finito, não abeliano. O subgrupo $V_{*}(K G)$ é normal em $V(K G)$ se e somente se $p=2$ e $G$ é o produto direto de um grupo abeliano elementar com um grupo $H$ para o qual vale uma das seguintes:

(i) H não possui fator direto de ordem 2, mas ele é o produto semidireto de um grupo $\langle b\rangle$ de ordem 2 e um 2-grupo abeliano A tal que $b^{-1} a b=a^{-1}$, para todo $a \in A$.

(ii) $H$ é um 2-grupo extra-especial ou o produto central de um tal grupo com um grupo cíclico de ordem 4 . 
Uma unidade $\alpha \in V(K G)$ diz-se simétrica se possui a propriedade que $\alpha^{*}=\alpha$. Ao contrário das unidades unitárias, em geral, as unidades simétricas não formam um subgrupo em $V(K G)$. V. Bovdi, L.G. Kovács e S.K. Sehgal [7] caracterizaram os grupos $G$ e os corpos $K$ para os quais o conjunto de unidades simétricas forma um subgrupo, com algumas hipóteses restritivas:

Teorema 1.3.6 Sejam p um primo, $K$ um anel comutativo de característica $p$, e $G$ um p-grupo localmente finito não-abeliano. As unidades simétricas de $K G$ formam um grupo multiplicativo se, e somente se, $p=2$ e $G$ é o produto direto de um grupo abeliano elementar e um grupo $H$ para o qual vale uma das seguintes:

(i) $H$ possui um subgrupo abeliano $A$ de indice 2 e um elemento $b$ de ordem 4 tal que $b^{-1} a b=a^{-1}$, para todo $a \in A ;$

(ii) H é o produto direto de um grupo quatérnio de ordem 8 e um grupo cíclico de ordem 4, ou o produto direto de dois grupos quatérnios de ordem 8;

(iii) $H$ é o produto central do grupo

$$
\left\langle x, y \mid x^{4}=y^{4}=1, x^{2}=[y, x]\right\rangle
$$

com um grupo quatérnio de ordem 8 , o elemento não-trivial comum aos dois grupos sendo $x^{2} y^{2}$;

(iv) $H$ é isomorfo a um dos grupos

$$
H_{32}=\left\langle x, y, u \mid x^{4}=y^{4}=1, x^{2}=[y, x], y^{2}=u^{2}=[u, x], x^{2} y^{2}=[u, y]\right\rangle
$$




$$
\begin{gathered}
H_{245}=\langle x, y, u, v| x^{4}=y^{4}=[v, u]=1, x^{2}=v^{2}=[y, x]=[v, y] \\
\left.y^{2}=u^{2}=[u, x], x^{2} y^{2}=[u, y]=[v, x]\right\rangle .
\end{gathered}
$$

Visto que $\left(\alpha^{*}\right)^{*}=\alpha$ e $(\alpha \beta)^{*}=\beta^{*} \alpha^{*}$, para todos $\alpha, \beta \in K G$, segue que todos os elementos da forma $\alpha \alpha^{*}$, com $\alpha \in V(K G)$, é uma unidade simétrica.

Observamos que se $V_{*}(K G)$ é normal em $V(K G)$, pelo Corolário 1.3.4, todo elemento da forma $x x^{*} \operatorname{com} x \in V(K G)$ é central em $K G$. Como cada unidade em $K G$ é um múltiplo escalar de uma unidade normalizada, a mesma conclusão é adequada se $x$ é uma unidade qualquer em $K G$. Deste modo, se $x \in U(K G)$, então

$$
x x^{*}=x^{-1}\left(x x^{*}\right) x=x^{*} x .
$$

Agora, se $x \notin U(K G)$, então $x \in \Delta(G)$ e disto $1+x$ é uma unidade e então vale

$$
(1+x)(1+x)^{*}=(1+x)^{*}(1+x)
$$

$\mathrm{e}$, conseqüentemente, novamente temos $x x^{*}=x^{*} x$.

Uma álgebra de grupo $K G$ na qual $x x^{*}=x^{*} x$ vale para cada elemento $x \in K G$ é chamada normal. A. Bovdi, P.M. Gudivok e M.S. Semirot provaram em [3] que a álgebra de grupo de um grupo não abeliano $G$ sobre um anel comutativo $K$ é normal se e somente se $G$ é hamiltoniano ou a característica de $K$ é 2 e $G$ é um produto direto de um 2-grupo abeliano elementar com um grupo $H$ tal que (i) ou (ii) do Teorema 1.3.5 vale. 


\section{Capítulo 2}

\section{A ordem do subgrupo unitário para característica $p>2$}

Neste capítulo determinamos a ordem do subgrupo unitário $V_{*}(K G)$ da álgebra de grupo $K G$ no caso em que a característica de $K$ é $p>2$ e $G$ é um $p$-grupo finito.

Ainda neste, determinamos a ordem do importante conjunto

$$
S_{*}(K G)=\left\{\alpha \in V(K G) \mid \alpha^{*}=\alpha\right\},
$$

o conjunto das unidades normalizadas simétricas da álgebra de grupo $K G$ para a característica $p>2$. 


\section{$2.1 \quad p$-grupos finitos $(p>2)$}

Sejam $K$ um corpo finito com $\operatorname{char}(K)=p>2$ e $G$ um $p$-grupo finito.

O conjunto

$$
\left\{\alpha=\sum_{g \in G} \alpha_{g} g \in K G \mid \varepsilon(\alpha)=\sum_{g \in G} \alpha_{g}=0\right\}
$$

é um ideal na álgebra de grupo $K G$, denotado por $\Delta(G)$ e chamado de ideal de aumento da álgebra $K G$. Isto é, $\Delta(G)$ é o conjunto de todos os elementos em $K G$ de aumento zero.

Considere

$$
S_{+}(\Delta(G))=\left\{\alpha \in \Delta(G) \mid \alpha^{*}=\alpha\right\}
$$

o conjunto de elementos simétricos de aumento zero em $K G \mathrm{e}$

$$
S_{-}(\Delta(G))=\left\{\alpha \in \Delta(G) \mid \alpha^{*}=-\alpha\right\}
$$

o conjunto de elementos anti-simétricos de aumento zero em $K G$.

Lema 2.1.1 Sejam $K$ um corpo com $\operatorname{char}(K)=p>2$ e $G$ um grupo de ordem ímpar. Então,

(i) $\Delta(G)=S_{+}(\Delta(G))+S_{-}(\Delta(G))$;

(ii) $S_{+}(\Delta(G)) \cap S_{-}(\Delta(G))=\{0\}$. 
Assim, $\Delta(G)=S_{+}(\Delta(G)) \oplus S_{-}(\Delta(G))$ como $K$-espaços.

Prova: (i) Seja $\alpha \in \Delta(G)$.

Tomemos $\beta=\frac{\alpha+\alpha^{*}}{2}$ e $\gamma=\frac{\alpha-\alpha^{*}}{2}$. Facilmente, vê-se que $\beta^{*}=\beta, \gamma^{*}=-\gamma$ e $\alpha=\beta+\gamma$, donde de conclui que $\Delta(G) \subset S_{+}(\Delta(G))+S_{-}(\Delta(G))$. Portanto, $\Delta(G)=S_{+}(\Delta(G))+$ $S_{-}(\Delta(G))$.

(ii) Seja $\alpha=\sum_{g \in G} \alpha_{g} g \in S_{+}(\Delta(G)) \cap S_{-}(\Delta(G))$. Então,

$$
\begin{gathered}
\alpha=\alpha^{*}=-\alpha \\
2 \alpha=0 \\
\alpha_{g}=0, \quad \forall g \in G,
\end{gathered}
$$

visto que $\operatorname{char}(K) \neq 2$.

Portanto, $\alpha=0$ e $S_{+}(\Delta(G)) \cap S_{-}(\Delta(G))=\{0\}$.

Corolário 2.1.2 Seja KG uma álgebra de grupo finita de característica $p>2$, então

$$
|\Delta(G)|=\left|S_{+}(\Delta(G))\right| \cdot\left|S_{-}(\Delta(G))\right|
$$

Exemplo 2.1.3 Considere a álgebra de grupo $\mathbf{Z}_{3} G$, onde $G=\left\langle a \mid a^{3}=1\right\rangle$. Temos então: 
(i) $\left|\mathbf{Z}_{3} G\right|=\left|\mathbf{Z}_{3}\right|^{|G|}=3^{3}=27$;

(ii) $\left|V\left(\mathbf{Z}_{3} G\right)\right|=\left|\mathbf{Z}_{3}\right|^{|G|-1}=3^{2}=9$;

(iii) $|\Delta(G)|=\left|V\left(\mathbf{Z}_{3} G\right)\right|=9$.

É fácil observarmos que

$$
\begin{aligned}
\Delta(G)= & \left\{\alpha=\sum_{i=0}^{2} \alpha_{i} a^{i} \in \mathbf{Z}_{3} G \mid \varepsilon(\alpha)=\sum_{i=0}^{2} \alpha_{i}=0\right\} \\
= & \left\{0,1+2 a, 1+2 a^{2}, 2+a, 2+a^{2}, a+2 a^{2},\right. \\
& \left.2 a+a^{2}, 1+a+a^{2}, 2+2 a+2 a^{2}\right\} .
\end{aligned}
$$

Assim,

$$
\begin{aligned}
S_{+}(\Delta(G)) & =\left\{\alpha \in \Delta(G) \mid \alpha^{*}=\alpha\right\} \\
& =\left\{0,1+a+a^{2}, 2+2 a+2 a^{2}\right\}
\end{aligned}
$$

e

$$
\begin{aligned}
S_{-}(\Delta(G)) & =\left\{\alpha \in \Delta(G) \mid \alpha^{*}=-\alpha\right\} \\
& =\left\{0,2 a+a^{2}, a+2 a^{2}\right\},
\end{aligned}
$$

onde se vê, $\left|S_{+}(\Delta(G))\right| \cdot\left|S_{-}(\Delta(G))\right|=|\Delta(G)|$ e $\Delta(G)=S_{+}(\Delta(G)) \oplus S_{-}(\Delta(G))$.

Lema 2.1.4 Sejam $K$ um corpo finito de característica $p>2$ e G um grupo finito de ordem ímpar. Então

$$
\left|S_{+}(\Delta(G))\right|=|K|^{\frac{|G|-1}{2}} .
$$


Prova: Seja $x=\sum_{g \in G} \alpha_{g} g \in S_{+}(\Delta(G))$. Visto que $x^{*}=x$, temos que

$$
\operatorname{supp}(x)=\operatorname{supp}\left(x^{*}\right) \text { e } \alpha_{g}=\alpha_{g^{-1}}, \forall g \in \operatorname{supp}(x) .
$$

Logo,

$$
x=\alpha_{1}+\sum_{\substack{g \in G \\ g \neq 1}} \alpha_{g}\left(g+g^{-1}\right) \operatorname{com} \sum_{g \in G} \alpha_{g} \equiv 0(\bmod p) .
$$

Mas,

$$
\varepsilon(x)=\alpha_{1}+2 \sum_{\substack{g \in G \\ g \neq 1}} \alpha_{g}=0
$$

isto é,

$$
\begin{gathered}
\alpha_{1}+2 \sum_{g \neq 1} \alpha_{g} \equiv 0(\bmod p) \\
\alpha_{1} \equiv-2 \sum_{g \neq 1} \alpha_{g}(\bmod p) .
\end{gathered}
$$

Portanto,

$$
\begin{aligned}
x & =\alpha_{1}+\sum_{\substack{g \in G \\
g \neq 1}} \alpha_{g}\left(g+g^{-1}\right) \\
& =-2 \sum_{\substack{g \in G \\
g \neq 1}} \alpha_{g}+\sum_{\substack{g \in G \\
g \neq 1}} \alpha_{g}\left(g+g^{-1}\right) \\
& =\sum_{\substack{g \in G \\
g \neq 1}} \alpha_{g}\left(g+g^{-1}-2\right) .
\end{aligned}
$$

Assim,

$$
S_{+}(\Delta(G))=\left\{x=\sum_{\substack{g \in G \\ g \neq 1}} \alpha_{g}\left(g+g^{-1}-2\right) \mid \alpha_{g} \in K\right\},
$$

donde se conclui que

$$
\left|S_{+}(\Delta(G))\right|=|K|^{\frac{|G|-1}{2}} .
$$


Exemplo 2.1.5 Considere a álgebra de grupo $\mathrm{Z}_{5} G$, onde $G=\left\langle a \mid a^{5}=1\right\rangle$. Temos pelo Lema 2.1.4 que $|S(\Delta(G))|=5^{2}=25$. A saber,

$$
\begin{aligned}
S(\Delta(G)) & =\left\{\alpha_{1}\left(a+a^{4}-2\right)+\alpha_{2}\left(a^{2}+a^{3}-2\right) \mid \alpha_{i} \in \mathbf{Z}_{5}, i=1,2\right\} \\
& =\left\{-2\left(\alpha_{1}+\alpha_{2}\right)+\alpha_{1}\left(a+a^{4}\right)+\alpha_{2}\left(a^{2}+a^{3}\right) \mid \alpha_{i} \in \mathbf{Z}_{5}, i=1,2\right\} \\
& =\left\{3\left(\alpha_{1}+\alpha_{2}\right)+\alpha_{1}\left(a+a^{4}\right)+\alpha_{2}\left(a^{2}+a^{3}\right) \mid \alpha_{i} \in \mathbf{Z}_{5}, i=1,2\right\} .
\end{aligned}
$$

Corolário 2.1.6 Sejam $K$ um corpo finito de característica $p>2$ e Gum p-grupo finito. Então

$$
\left|S_{-}(\Delta(G))\right|=|K|^{\frac{|G|-1}{2}} \text {. }
$$

Corolário 2.1.7 Sejam $K$ um corpo finito de característica $p>2$ e G um p-grupo finito. Então

$$
\left|S_{*}(K G)\right|=|K|^{\frac{|G|-1}{2}} \text {. }
$$

Prova: Seja $\alpha=\sum_{g \in G} \alpha_{g} g \in S_{*}(K G)$. Logo $\alpha^{*}=\alpha$ e $\varepsilon(\alpha)=\sum_{g \in G} \alpha_{g}=1$. Assim,

$$
\begin{gathered}
\operatorname{supp}\left(\alpha^{*}\right)=\operatorname{supp}(\alpha) \\
\alpha_{g}=\alpha_{g^{-1}}, \quad \forall g \in \operatorname{supp}(\alpha) \quad \text { e } \sum_{g \in G} \alpha_{g}=1 .
\end{gathered}
$$

Assim, $\alpha=\alpha_{1}+\sum_{\substack{g \in G \\ g \neq 1}} \alpha_{g}\left(g+g^{-1}\right)$. 
Logo,

$$
\begin{aligned}
\varepsilon(\alpha) & =\alpha_{1}+\sum_{g \neq 1} 2 \alpha_{g}=1 \\
\alpha_{1} & \equiv 1-2 \sum_{g \neq 1} \alpha_{g}(\bmod p) .
\end{aligned}
$$

Deste modo,

$$
\begin{aligned}
& \alpha=\left(1-2 \sum_{\substack{g \in G \\
g \neq 1}} \alpha_{g}\right)+\sum_{\substack{g \in G \\
g \neq 1}} \alpha_{g}\left(g+g^{-1}\right) \\
& \alpha=1+\sum_{\substack{g \in G \\
g \neq 1}} \alpha_{g}\left(g+g^{-1}-2\right)
\end{aligned}
$$

e então

$$
\left|S_{*}(K G)\right|=|K|^{\frac{|G|-1}{2}} .
$$

$\mathrm{Na}$ realidade, observamos que

$$
S_{*}(K G)=1+S_{+}(\Delta(G))
$$

Teorema 2.1.8 Sejam $K$ um corpo finito de característica $p>2$ e G um p-grupo finito. Então

$$
\left|V_{*}(K G)\right|=|K|^{\frac{|G|-1}{2}}
$$

Prova: Observemos que, se $x \in S_{-}(\Delta(G))$, então visto que $U(K G)=\{\alpha \in K G \mid \varepsilon(\alpha) \neq$ $0\}$, o elemento $1+x$ é uma unidade em $K G$ e, pelo Lema $1.2 .2, u=(1-x)(1+x)^{-1}$ é uma unidade unitária de Cayley. 
É fácil observarmos que qualquer elemento em $V_{*}(K G)$ é uma unidade unitária de Cayley para $\operatorname{char}(K)>2$.

De fato, se $u \in V_{*}(K G)$ então $\varepsilon(u)=1$ e $1+u$ é uma unidade, visto que

$$
U(K G)=\{\alpha \in K G \mid \varepsilon(\alpha) \neq 0\}
$$

Assim, $k=(1-u)(1+u)^{-1}$ é um elemento anti-simétrico em $K G$. Logo, $1+k=$ $2(1+u)^{-1}$ é uma unidade em $K G$, e $u=(1-k)(1+k)^{-1}$ é uma unidade unitária de Cayley.

Portanto,

$$
\left|V_{*}(K G)\right|=\left|S_{-}(\Delta(G))\right|=|K|^{\frac{|G|-1}{2}} .
$$




\section{Capítulo 3}

\section{A ordem do subgrupo unitário para alguns produtos centrais}

Neste capítulo determinamos a ordem do subgrupo unitário $V_{*}(K G)$ da álgebra de grupo $K G$ quando $K$ é um corpo finito de característica 2 e $G$ é um 2-grupo extraespecial ou um produto central de um 2-grupo extra-especial com um grupo cíclico de ordem 4. 


\section{$3.1 \quad 2$-grupo extra-especial}

Para uma álgebra de grupo $K G$, denotamos $S_{K}(G)=\left\{x x^{*} \mid x \in V(K G)\right\}$ que é, como já visto, um conjunto de unidades simétricas em $K G$.

Lema 3.1.1 Se $V_{*}(K G)$ é normal em $V(K G)$, então $S_{K}(G)$ é um subgrupo normal em $V(K G)$

Prova: Sendo $V_{*}(K G) \triangleleft V(K G)$, segue que a álgebra $K G$ é normal, i.e, $x x^{*} \doteq x^{*} x$, para todo $x \in K G$, pela conclusão do capítulo 1. Pelo Corolário 1.3.4, temos também que $x x^{*}$ é central em $K G$ sempre que $x \in V(K G)$. Logo,nós temos

$$
\begin{aligned}
\left(x x^{*}\right)\left(y y^{*}\right)^{-1} & =x x^{*}\left(y^{*}\right)^{-1} y^{-1}=x x^{*}\left(y^{-1}\right)^{*} y^{-1} \\
& =\left(y^{-1}\right)^{*} x x^{*} y^{-1}=\left(y^{-1}\right)^{*} x^{*} x y^{-1}=\left(x y^{-1}\right)^{*}\left(x y^{-1}\right) \\
& =\left(x y^{-1}\right)\left(x y^{-1}\right)^{*},
\end{aligned}
$$

para todos $x, y$ em $V(K G)$, o que nos diz que $S_{K}(G)$ é um subgrupo em $V(K G)$. A normalidade de $S_{K}(G)$ segue do fato que cada elemento de $S_{K}(G)$ é estável por conjugação em $V(K G)$.

Lema 3.1.2 Sejam K um corpo de característica 2 e G um 2-grupo extra-especial. Então a aplicação $x \mapsto x x^{*}$ é um epimorfismo do grupo $V(K G)$ no subgrupo $S_{K}(G)$. Além disso, se $V(K G)$ é finito, então a ordem do subgrupo unitário $V_{*}(K G)$ coincide com o índice do subgrupo $S_{K}(G)$ em $V(K G)$. 
Prova: Pelo Teorema 1.3.5 e Corolário 1.3.4, temos que $x x^{*}$ é central em $V(K G)$ para todo $x \in V(K G)$. Fazendo $\phi(x)=x x^{*}$, temos

$$
\phi(x y)=(x y)(x y)^{*}=x y y^{*} x^{*}=x x^{*} y y^{*}=\phi(x) \phi(y)
$$

para todos $x, y \in V(K G)$. Portanto, $\phi$ é um epimorfismo. Agora, o núcleo de $\phi$ é

$$
\left\{x \in V(K G) \mid x x^{*}=1\right\}
$$

facilmente visto ser $V_{*}(K G)$, donde segue o resto do Lema.

Para um 2-grupo finito $G$ com subgrupo comutador $G^{\prime}=\left\langle c \mid c^{2}=1\right\rangle$, definimos $L_{G}$ como sendo um subconjunto de elementos de ordem $4 \mathrm{em} G$ tal que $L_{G} \cap L_{G} c$ é vazio e $L_{G} \cup L_{G} c$ coincide com o conjunto de todos os elementos de ordem $4 \mathrm{em} G$.

Lema 3.1.3 Seja $G$ um 2-grupo extra-especial de ordem $|G|=2^{2 n+1}$, com $n \geq 2$. Então

$$
\left|L_{G}\right|=2^{n-1}\left(2^{n}-(-1)^{n}\right) .
$$

Prova: Sendo $G$ um 2-grupo extra-especial de ordem $|G|=2^{2 n+1}$, com $n \geq 2$, então, pelo Corolário 1.1.8, nós temos $G=G_{1} \bigvee \ldots \bigvee_{G_{n}}$, onde $G_{i}$ é um grupo quatérnio de ordem 8 . Então, $Z(G)=G^{\prime}=\left\langle c \mid c^{2}=1\right\rangle$ e $G_{i} \cap G_{j}=\langle c\rangle$ para quaisquer $i \neq j$. Evidentemente cada elemento de ordem 4 de $G$ pode ser escrito como

$$
x=z_{i_{1}} z_{i_{2}} \ldots z_{i_{s}}
$$


onde $z_{i_{k}} \in G_{i_{k}}$ tem ordem 4 e $i_{1}<i_{2}<\cdots<i_{s}$. Chamamos $s$ o comprimento de $x$. Pelo fato que $Z(G)=G^{\prime}$ e do Lema 1.1.3, nós temos que $z_{i_{k}}^{2}=c$ e $x^{2}=z_{i_{1}}^{2} z_{i_{2}}^{2} \cdots z_{i_{s}}^{2}=c^{s}$. Sendo a ordem de $x$ igual a 4 , devemos ter $s \equiv 1(\bmod 2)$, i.e, $s$ deve ser ímpar.

Considere $H_{k}=H\left(i_{1}, i_{2}, \ldots, i_{k}\right)=G_{i_{1}} Y_{G_{i_{2}}} Y \ldots Y_{G_{i_{k}}}$, onde $k$ é ímpar e $i_{1}<i_{2}<\cdots<$ $i_{k}$. Nosso objetivo aqui é mostrar que existem precisamente $3^{k}$ elementos de comprimento $k$ em $L_{H_{k}}$. Cada $L_{G_{i}}$ contém três diferentes elementos e cada elemento de comprimento $k$ da forma (1) tem ordem 4. Portanto, o número de elementos de $L_{H_{k}}$ é $3^{k}$. Agora, o número de diferentes subgrupos $H_{k}$ de $G$ é $\left(\begin{array}{l}n \\ k\end{array}\right)$ e, então, o número de elementos de $L_{G}$ é

$$
\left|L_{G}\right|=\sum_{j=1}^{m}\left(\begin{array}{c}
n \\
2 j-1
\end{array}\right) 3^{2 j-1},
$$

onde $m=\frac{n+1}{2}$ se $n$ é ímpar e $m=\frac{n}{2}$ se $n$ é par.

Se escrevemos

$$
M_{1}=2^{2 n}=(1+3)^{n}=\sum_{i=0}^{n}\left(\begin{array}{c}
n \\
i
\end{array}\right) 3^{i}
$$

e

$$
M_{2}=(-1)^{n} 2^{n}=(1-3)^{n}=\sum_{i=0}^{n}\left(\begin{array}{c}
n \\
i
\end{array}\right)(-1)^{i} 3^{i},
$$

então podemos obter

$$
\begin{aligned}
\left|L_{G}\right| & =\sum_{j=1}^{m}\left(\begin{array}{c}
n \\
2 j-1
\end{array}\right) 3^{2 j-1}=\frac{1}{2}\left(\sum_{i=0}^{n}\left(\begin{array}{c}
n \\
i
\end{array}\right) 3^{i}-\sum_{i=0}^{n}\left(\begin{array}{c}
n \\
i
\end{array}\right)(-1)^{i} 3^{i}\right) \\
& =\frac{1}{2}\left(M_{1}-M_{2}\right)=2^{n-1}\left(2^{n}-(-1)^{n}\right) .
\end{aligned}
$$


Lema 3.1.4 Sejam $K$ um corpo finito de característica 2 e $G$ um 2-grupo extra-especial de ordem $|G|=2^{2 n+1}$, com $n \geq 2$. Então, para todo $x \in V(K G)$,

$$
x x^{*}=\prod_{b \in L_{G}}\left(1+\alpha_{b} b(1+c)\right)
$$

onde $\alpha_{b} \in K$.

Prova: Seja $x=\sum_{i=1}^{t} \alpha_{i} a_{i} \in V(K G)$. Então $x^{*}=\sum_{i=1}^{t} \alpha_{i} a_{i}^{-1} \mathrm{e}$

$$
\begin{aligned}
x x^{*} & =\left(\alpha_{1} a_{1}+\cdots+\alpha_{t} a_{t}\right)\left(\alpha_{1} a_{1}^{-1}+\cdots+\alpha_{t} a_{t}^{-1}\right) \\
& =\left(\alpha_{1}^{2}+\cdots+\alpha_{t}^{2}\right)+\sum_{\substack{i, j \\
i<j}} \alpha_{i} \alpha_{j}\left(a_{i} a_{j}^{-1}+a_{j} a_{i}^{-1}\right) .
\end{aligned}
$$

Temos que analisar dois casos sobre a ordem de $a_{i} a_{j}^{-1}$ :

Se a ordem de $a_{i} a_{j}^{-1}$ é 2 , então $a_{i} a_{j}^{-1}=\left(a_{i} a_{j}^{-1}\right)^{-1}=a_{j} a_{i}^{-1}$. Assim, o suporte de $x x^{*}$ não contém elementos de ordem 2.

Portanto, a ordem de $a_{i} a_{j}^{-1}$ deve ser 4. Visto que $g^{2} \in G^{\prime}=\left\langle c \mid c^{2}=1\right\rangle$, para todo $g \in$ $G$, devemos ter $\left(a_{i} a_{j}^{-1}\right)^{2}=c$ e disto $a_{i} a_{j}^{-1}=c a_{j} a_{i}^{-1}$. Como $\varepsilon(x)=\sum_{i=1}^{t} \alpha_{i}=1 \mathrm{e}$ característica de $K$ é 2 , segue que

$$
x x^{*}=1+\sum_{\substack{i, j \\ i<j}} \alpha_{i j} a_{i} a_{j}^{-1}(1+c),
$$

com $a_{i} a_{j}^{-1} \in L_{G}$. Denotando $a_{i} a_{j}^{-1}$ por $b$ e observando que $(1+c)^{2}=0$, segue que

$$
x x^{*}=1+\sum_{b \in L_{G}} \alpha_{b} b(1+c)=\prod_{b \in L_{G}}\left(1+\alpha_{b} b(1+c)\right)
$$


Teorema 3.1.5 Sejam $K$ um corpo finito de característica 2 e $G$ um 2-grupo extraespecial de ordem $|G|=2^{2 n+1}$, com $n \geq 2$. Então

$$
\left|V_{*}(K G)\right|=|K|^{2^{n-1}\left(2^{n+2}-2^{n}+(-1)^{n}\right)-1} .
$$

Prova: Vemos claramente pelo Lema 3.1.4 que a ordem de $S_{K}(G)=\left\{x x^{*} \mid x \in V(K G)\right\}$ é no máximo $|K|^{\left|L_{G}\right|}$. Nosso objetivo aqui é provar que $\left|S_{K}(G)\right|$ é exatamente $|K|^{\left|L_{G}\right|}$.

Tome $b \in L_{G}$ arbitrário e um $\alpha_{b} \in K$. Então existe um elemento $\omega_{b} \in G$ de ordem 2 tal que $\left[\omega_{b}, b\right] \neq 1$. De fato, sendo $b=z_{i_{1}} z_{i_{2}} \cdots z_{i_{2 k+1}} \in L_{G}$, então escolhemos um $u_{1} \in G_{i_{1}}$ de ordem 4 tal que $\left[u_{1}, z_{i_{1}}\right] \neq 1$ e um elemento $u_{2}$ do conjunto $\left\{z_{i_{2}}, \ldots, z_{i_{2 k+1}}\right\}$ se $k \neq 0$, e caso $k=0$, escolhemos $u_{2}$ de $G_{t}$ para algum $t \neq i_{1} \operatorname{com}\left|u_{2}\right|=4$. Assim, $\omega_{b}=u_{1} u_{2}$ é um elemento de ordem 2 em $G$ tal que $\left[\omega_{b}, b\right] \neq 1$.

Agora,

$$
\begin{aligned}
\left(1+\alpha_{b}\left(b+\omega_{b}\right)\right) \cdot\left(1+\alpha_{b}\left(b+\omega_{b}\right)\right)^{*}= & \left(1+\alpha_{b}\left(b+\omega_{b}\right)\right) \cdot\left(1+\alpha_{b}\left(b^{-1}+\omega_{b}^{-1}\right)\right. \\
= & \left(1+\alpha_{b}\left(b+\omega_{b}\right)\right) \cdot\left(1+\alpha_{b}\left(c b+\omega_{b}\right)\right. \\
= & 1+\alpha_{b}\left(c b+\omega_{b}\right)+\alpha_{b}\left(b+\omega_{b}\right) \\
& +\alpha_{b}^{2}\left(b+\omega_{b}\right)\left(c b+\omega_{b}\right) \\
= & 1+\alpha_{b} b(1+c)+\alpha_{b}^{2}\left(1+b \omega_{b}+c \omega_{b} b+1\right) \\
= & 1+\alpha_{b} b(1+c)+\alpha_{b}^{2}\left(b \omega_{b}+b \omega_{b}\right) \\
= & 1+\alpha_{b} b(1+c),
\end{aligned}
$$


que nos mostra que qualquer fator do produto $\prod_{b \in L_{G}}\left(1+\alpha_{b} b(1+c)\right)$ pertence ao subgrupo $S_{K}(G)$.

Como $u u^{*}$ é central em $K G$ para qualquer $u \in V(K G)$, conforme Corolário 1.3.4, temos ainda que

$$
\begin{aligned}
& \prod_{b \in L_{G}}\left(1+\alpha_{b}\left(b+\omega_{b}\right)\right)\left(1+\alpha_{b}\left(b+\omega_{b}\right)\right)^{*}= \\
&=\left(1+\alpha_{b_{1}}\left(b_{1}+\omega_{b_{1}}\right)\right)\left(1+\alpha_{b_{1}}\left(b_{1}+\omega_{b_{1}}\right)\right)^{*}\left(1+\alpha_{b_{2}}\left(b_{2}+\omega_{b_{2}}\right)\right)\left(1+\alpha_{b_{2}}\left(b_{2}+\omega_{b_{2}}\right)\right)^{*} \cdots \\
& \cdots\left(1+\alpha_{b_{t}}\left(b_{t}+\omega_{b_{t}}\right)\right)\left(1+\alpha_{b_{t}}\left(b_{t}+\omega_{b_{t}}\right)\right)^{*} \\
&=\left(1+\alpha_{b_{1}}\left(b_{1}+\omega_{b_{1}}\right)\right)\left(1+\alpha_{b_{2}}\left(b_{2}+\omega_{b_{2}}\right)\right)\left(1+\alpha_{b_{2}}\left(b_{2}+\omega_{b_{2}}\right)\right)^{*}\left(1+\alpha_{b_{1}}\left(b_{1}+\omega_{b_{1}}\right)\right)^{*} \cdots \\
& \cdots\left(1+\alpha_{b_{t}}\left(b_{t}+\omega_{b_{t}}\right)\right)\left(1+\alpha_{b_{t}}\left(b_{t}+\omega_{b_{t}}\right)\right)^{*} \\
&=\left(1+\alpha_{b_{1}}\left(b_{1}+\omega_{b_{1}}\right)\right)\left(1+\alpha_{b_{2}}\left(b_{2}+\omega_{b_{2}}\right)\right)\left(1+\alpha_{b_{3}}\left(b_{3}+\omega_{b_{3}}\right)\right)\left(1+\alpha_{b_{3}}\left(b_{3}+\omega_{b_{3}}\right)\right)^{*} \cdots \\
& \cdots\left(1+\alpha_{b_{t}}\left(b_{t}+\omega_{b_{t}}\right)\right)\left(1+\alpha_{b_{t}}\left(b_{t}+\omega_{b_{t}}\right)\right)^{*}
\end{aligned}
$$

assim, observando que

$$
\left(u_{1} u_{2} \cdots u_{n} u_{n}^{*} \cdots u_{2}^{*} u_{1}^{*}\right) u_{n+1} u_{n+1}^{*}=u_{1} u_{2} \cdots u_{n} u_{n+1} u_{n+1}^{*} u_{n}^{*} \cdots u_{2}^{*} u_{1}^{*}, \forall n
$$

temos que após $(t-1)$ passos obtemos,

$$
\begin{aligned}
\prod_{b \in L_{G}}\left(1+\alpha_{b}\left(b+\omega_{b}\right)\right)\left(1+\alpha_{b}\left(b+\omega_{b}\right)\right)^{*}= \\
=\left(1+\alpha_{b_{1}}\left(b_{1}+\omega_{b_{1}}\right)\right)\left(1+\alpha_{b_{2}}\left(b_{2}+\omega_{b_{2}}\right)\right) \cdots\left(1+\alpha_{b_{t}}\left(b_{t}+\omega_{b_{t}}\right)\right)\left(1+\alpha_{b_{t}}\left(b_{t}+\omega_{b_{t}}\right)\right)^{*} \cdots \\
\quad \cdots\left(1+\alpha_{b_{2}}\left(b_{2}+\omega_{b_{2}}\right)\right)^{*}\left(1+\alpha_{b_{1}}\left(b_{1}+\omega_{b_{1}}\right)\right)^{*} \\
=\left(1+\alpha_{b_{1}}\left(b_{1}+\omega_{b_{1}}\right)\right)\left(1+\alpha_{b_{2}}\left(b_{2}+\omega_{b_{2}}\right)\right) \cdots\left(1+\alpha_{b_{t}}\left(b_{t}+\omega_{b_{t}}\right)\right) . \\
\quad\left(\left(1+\alpha_{b_{1}}\left(b_{1}+\omega_{b_{1}}\right)\right)\left(1+\alpha_{b_{2}}\left(b_{2}+\omega_{b_{2}}\right)\right) \cdots\left(1+\alpha_{b_{t}}\left(b_{t}+\omega_{b_{t}}\right)\right)\right)^{*} \\
=\prod_{b \in L_{G}}\left(1+\alpha_{b}\left(b+\omega_{b}\right)\right) .\left(\prod_{b \in L_{G}}\left(1+\alpha_{b}\left(b+\omega_{b}\right)\right)\right)^{*},
\end{aligned}
$$


o que nos mostra que $\prod_{b \in L_{G}}\left(1+\alpha_{b}\left(b+\omega_{b}\right)\right)\left(1+\alpha_{b}\left(b+\omega_{b}\right)\right)^{*}$ pertence ao subgrupo $S_{K}(G)$.

Portanto, temos que $\left|S_{K}(G)\right|=|K|^{\left|L_{G}\right|}$. Agora, pelo Lema 3.1.2, temos que $\left|V_{*}(K G)\right|=$ $|V(K G)| /\left|S_{K}(G)\right|$. Como $|V(K G)|=|K|^{|G|-1}=|K|^{2^{2 n+1}-1}$ e, pelo Lema 3.1.3, vemos que $\left|L_{G}\right|=2^{n-1}\left(2^{n}-(-1)^{n}\right)$, segue que

$$
\begin{aligned}
\left|V_{*}(K G)\right| & =|K|^{2^{2 n+1}-1} \cdot|K|^{-2^{n-1}\left(2^{n}-(-1)^{n}\right)} \\
& =|K|^{2^{n-1}\left(2^{n+2}-2^{n}+(-1)^{n}\right)-1} .
\end{aligned}
$$

\subsection{Produto central de um 2-grupo extra-especial com um grupo cíclico de ordem 4}

Neste parágrafo determinamos a ordem do subgrupo unitário $V_{*}(K G)$ quando $K$ é um corpo finito de característica 2 e $G=H Y_{C_{4}}$, onde $H$ é um 2-grupo extra-especial de ordem $|H|=2^{2 n+1}$ e $C_{4}=\langle d\rangle$ é um grupo cíclico de ordem 4. Uma vez já analisado o caso em que $G$ é um 2-grupo extra-especial, torna-se de fácil análise esta nova situação.

Lema 3.2.1 Sejam $K$ um corpo finito de característica 2 e $G$ um produto central de um 2-grupo extra-especial $H$ de ordem $|H|=2^{2 n+1}$ com um grupo cíclico $\langle d\rangle$ de ordem 4 . Então a aplicação $x \mapsto x x^{*}$ é um epimorfismo do grupo $V(K G)$ no subgrupo $S_{K}(G)$. Além disso, se $V(K G)$ é finito, então a ordem do subgrupo unitário $V_{*}(K G)$ coincide com o índice do subgrupo $S_{K}(G)$ em $V(K G)$. 
Prova: É só observarmos que, pelo Teorema 1.3.5 e Corolário 1.3 .4 , nós temos que $x x^{*}$ é central em $V(K G)$ para todo $x \in V(K G)$. A conclusão deste Lema segue análoga à do Lema 3.1.2.

Lema 3.2.2 Seja $G$ um produto central de um 2-grupo extra-especial $H$ de ordem $|H|=$ $2^{2 n+1}$ com um grupo cíclico $\langle d\rangle$ de ordem 4. Então

$$
\left|L_{G}\right|=2^{2 n} .
$$

Prova: Sendo $G=H Y\langle d\rangle$ com $H$ um 2-grupo extra-especial de ordem $2^{2 n+1}$ e $\langle d\rangle$ de ordem 4, temos identificado que $d^{2}=c$, onde $G^{\prime}=Z(G)=\left\langle c \mid c^{2}=1\right\rangle$. Logo, visto que $H=H_{1} \bigvee \ldots Y_{H_{n}}$, com $H_{i} \cong Q_{8}, 1 \leq i \leq n$, temos que cada elemento de ordem 4 em $G$ pode ser escrito como

$$
x=z_{i_{1}} z_{i_{2}} \cdots z_{i_{s}} d^{k}
$$

onde $z_{i_{t}} \in H_{i_{t}}$ tem ordem 4, com $i_{1}<\cdots<i_{s}$ e $k=0,1$. Como antes observamos no Lema 3.1.3, visto que ordem de $x$ é 4 e $x^{2}=z_{i_{1}}^{2} z_{i_{2}}^{2} \cdots z_{i_{s}}^{2} d^{2 k}=c^{s+k}$, devemos ter $k=0$ e $s$ ímpar ou que $k=1$ e $s$ par. Deste modo, concluímos que qualquer elemento de ordem 4 em $G$ ou pertence a $H$ ou pode ser escrito como $h d$, onde $h \in H$ e $|h| \neq 4$. O número de elemento de ordem 4 exclusivamente de $H$ é igual a $\left|L_{H}\right|$. Agora, o número de elementos $h \in H \operatorname{com}|h| \neq 4$ é igual a $|H|-2\left|L_{H}\right|$. Portanto, concluímos que

$$
\left|L_{G}\right|=\left|L_{H}\right|+\frac{|H|-2\left|L_{H}\right|}{2}=\frac{|H|}{2}=2^{2 n} .
$$


Teorema 3.2.3 Sejam $K$ um corpo finito de característica 2 e $G$ um produto central de um 2-grupo extra-especial $H$ de ordem $|H|=2^{2 n+1}$ com um grupo cíclico $\langle d\rangle$ de ordem 4 . Então

$$
\left|V_{*}(K G)\right|=|K|^{3.2^{2^{n}}-1} \text {. }
$$

Prova: De forma análoga à análise feita no Lema 3.1.4, vê-se que para todo $x \in V(K G)$, $\operatorname{com} G=H Y\langle d\rangle$, temos que

$$
x x^{*}=1+\sum_{b \in L_{G}} \alpha_{b} b(1+c)=\prod_{b \in L_{G}}\left(1+\alpha_{b} b(1+c)\right) .
$$

Como $H=H_{1} \bigvee \ldots Y_{H_{n}}$, onde $H_{i} \cong Q_{8}$ e $1 \leq i \leq n$, é um 2-grupo extra-especial de ordem $|H|=2^{2 n+1}$, pela prova do Teorema 3.1 .5 vemos que $1+\alpha_{b} b(1+c) \in S_{K}(G)$, para todo $b \in L_{H}$, e por conseguinte $1+\sum_{b \in L_{G}} \alpha_{b} b(1+c) \in S_{K}(G)$. Portanto, nós temos que

$$
|K|^{\left|L_{H}\right|} \leq\left|S_{K}(G)\right| \leq|K|^{\left|L_{G}\right|}
$$

Objetivamos aqui mostrar que $\left|S_{K}(G)\right|=|K|^{\left|L_{G}\right|}$. Para isto, devemos mostrar que $1+\alpha_{h d} h d(1+c) \in S_{K}(G) \operatorname{com} h \in H$ e $|h| \neq 4$.

Seja dado o elemento $h d \in G \operatorname{com} h \in H$ e $|h| \neq 4$. Obviamente, temos que $h d \in L_{G}$. 
Se $h=1$, então tomando $x=1+a_{i}+d$ e $y=1+a_{i}+b_{i} d$, que são unidades evidentemente, onde $H_{i}=\left\langle a_{i}, b_{i}\right\rangle$ para algum $i \in\{1,2, \ldots, n\}$, obtemos então que

$$
\begin{aligned}
x x^{*} & =\left(1+a_{i}+d\right)\left(1+a_{i}+d\right)^{*}=\left(1+a_{i}+d\right)\left(1+a_{i}^{-1}+d^{-1}\right) \\
& =\left(1+a_{i}+d\right)\left(1+a_{i} c+d c\right) \\
& =1+a_{i} c+d c+a_{i}+1+a_{i} d c+d+d a_{i} c+1 \\
& =1+\left(a_{i}+d\right)(1+c)
\end{aligned}
$$

e

$$
\begin{aligned}
y y^{*} & =\left(1+a_{i}+b_{i} d\right)\left(1+a_{i}+b_{i} d\right)^{*}=\left(1+a_{i}+b_{i} d\right)\left(1+a_{i}^{-1}+d^{-1} b_{i}^{-1}\right) \\
& =\left(1+a_{i}+b_{i} d\right)\left(1+a_{i} c+b_{i} d\right) \\
& =1+a_{i} c+b_{i} d+a_{i}+1+a_{i} b_{i} d+b_{i} d+b_{i} a_{i} d c+1 \\
& =1+a_{i}(1+c)
\end{aligned}
$$

visto que $a_{i}^{b_{i}}=a_{i}^{-1}=a_{i} c,\left[H_{i},\langle d\rangle\right]=1$ e $g^{2} \in G^{\prime}=Z(G)=\left\langle c \mid c^{2}=1\right\rangle$, para todo $g \in G$.

Portanto, obtemos que

$$
\begin{aligned}
1+d(1+c) & =1+\left(a_{i}+d+a_{i}\right)(1+c) \\
& =\left(1+\left(a_{i}+d\right)(1+c)\right)\left(1+a_{i}(1+c)\right) \\
& =\left(x x^{*}\right)\left(y y^{*}\right) \\
& =x y y^{*} x^{*}=(x y)(x y)^{*} \in S_{K}(G)
\end{aligned}
$$

visto que, pelo Teorema 1.3.5 e Corolário 1.3.4, temos que $y y^{*}$ é central em $K G$. 
Agora, se $|h|=2$, então $h$ pode ser escrito como

$$
h=z_{i_{1}} z_{i_{2}} \cdots z_{i_{2 k}}
$$

onde $z_{i_{t}} \in H_{i_{t}}$ tem ordem 4 , com $i_{1}<\cdots<i_{2 k}$. Neste caso, basta-nos tomar $x=$ $1+z_{i_{1}}+h d$ e $y=1+z_{i_{1}}+u_{1} h d$, onde $u_{1}$ é um outro elemento de $H_{i_{1}}$ de ordém 4 e tal que $\left[u_{1}, z_{i_{1}}\right] \neq 1$. É óbvio que a ordem de $u_{1} h d$ é 2 . Assim, temos que

$$
\begin{aligned}
x x^{*} & =\left(1+z_{i_{1}}+h d\right)\left(1+z_{i_{1}}+h d\right)^{*} \\
& =\left(1+z_{i_{1}}+h d\right)\left(1+z_{i_{1}}^{-1}+(h d)^{-1}\right) \\
& =\left(1+z_{i_{1}}+h d\right)\left(1+z_{i_{1}} c+h d c\right) \\
& =1+z_{i_{1}} c+h d c+z_{i_{1}}+1+z_{i_{1}} h d c+h d+h z_{i_{1}} d c+1 \\
& =1+\left(z_{i_{1}}+h d\right)(1+c)
\end{aligned}
$$

e

$$
\begin{aligned}
y y^{*} & =\left(1+z_{i_{1}}+u_{1} h d\right)\left(1+z_{i_{1}}+u_{1} h d\right)^{*} \\
& =\left(1+z_{i_{1}}+u_{1} h d\right)\left(1+z_{i_{1}}^{-1}+\left(u_{1} h d\right)^{-1}\right) \\
& =\left(1+z_{i_{1}}+u_{1} h d\right)\left(1+z_{i_{1}} c+u_{1} h d\right) \\
& =1+z_{i_{1}} c+u_{1} h d+z_{i_{1}}+1+z_{i_{1}} u_{1} h d+u_{1} h d+u_{1} z_{i_{1}} h d c+1 \\
& =1+z_{i_{1}}(1+c),
\end{aligned}
$$

visto que, se $\left[u_{1}, z_{i_{1}}\right] \neq 1$, então $\left[u_{1}, z_{i_{1}}\right]=c$, pois $G^{\prime}=\left\langle c \mid c^{2}=1\right\rangle$. Da mesma forma que acima, nós temos

$$
1+h d(1+c)=x x^{*} y y^{*}=(x y)(x y)^{*} \in S_{K}(G) .
$$


Deste modo, concluímos que $\left|S_{K}(G)\right|=|K|^{\left|L_{G}\right|}$. Visto que $|V(K G)|=|K|^{|G|-1}=$ $|K|^{2 n+2}-1$, pelo Lema 3.2.1 e Lema 3.2.2 obtemos que

$$
\left|V_{*}(K G)\right|=|K|^{2^{2 n+2}-1} \cdot|K|^{-2^{2 n}}=|K|^{2^{2 n}\left(2^{2}-1\right)-1}=|K|^{3 \cdot 2^{2 n}-1} .
$$




\section{Capítulo 4}

\section{A ordem do subgrupo unitário para os 2-grupos diedrais e para os quatérnios generalizados}

Neste capítulo determinamos a ordem do subgrupo unitário $V_{*}(K G)$ quando $K$ é um corpo finito de característica 2 e $G$ é um produto semidireto de um 2-grupo abeliano $A$ de índice 2 com um grupo $\langle b\rangle$ tal que $b$ inverte cada elemento de $A$, i.e, $a^{b}=a^{-1}$, para todo $a \in A$.

Observamos que sendo $A$ um subgrupo abeliano de índice $2 \mathrm{em} G$, então $G=A \cup b A$ e disto $b^{2} \in A$. Logo, $b^{2}=b^{-1} b^{2} b=b^{-2}$ e disto $b^{4}=1$. Portanto, a ordem de $b$ só pode ser 2 ou 4.

Se ordem de $b$ é 2 , inclui-se neste caso os 2-grupos diedrais e se ordem de $b$ é 4 , inclui-se 
então os quatérnios generalizados.

\section{$4.1 \quad 2$-grupos diedrais}

Lema 4.1.1 Sejam $K$ um corpo de característica 2 e $G$ um 2-grupo contendo um subgrupo abeliano $A$ de índice 2 e um elemento $b$ que inverte cada elemento de $A$. Então a aplicação $x \mapsto x x^{*}$ é um epimorfismo do grupo $V(K G)$ no subgrupo $S_{K}(G)$. Além disso, se $V(K G)$ é finito, então a ordem do subgrupo unitário $V_{*}(K G)$ coincide com o índice do subgrupo $S_{K}(G)$ em $V(K G)$.

Prova: Análoga à prova do Lema 3.1.2.

Para o grupo abeliano $A$, definimos $A[2]=\left\{a \in A \mid a^{2}=1\right\}$.

Lema 4.1.2 Sejam $K$ um corpo finito de característica 2 e $G$ um 2-grupo contendo um subgrupo abeliano $A$ de indice 2 e um elemento $b$ de ordem 2 que inverte cada elemento de A. Então, para todo $x \in V(K G)$, nós temos que

$$
x x^{*}=1+\sum_{a \in L} \alpha_{a}\left(a+a^{-1}\right)
$$

onde $L$ é um sistema completo de representantes do subconjunto $\left\{a^{-1}, a \mid a \in A \backslash A[2]\right\}$ e $\alpha_{a} \in K$ para todo $a \in A$. 
Prova: Qualquer elemento $x \in V(K G)$ pode ser escrito como $x=x_{0}+x_{1} b$, onde $x_{i} \in K A$, $i=0,1$, e $\varepsilon\left(x_{0}\right)+\varepsilon\left(x_{1}\right)=1$. Pelo fato que $a^{b}=a^{-1}$, para todo $a \in A$, segue imediatamente que $x_{i}^{b}=x_{i}^{*}, i=0,1$. Logo, temos que

$$
\begin{aligned}
x x^{*} & =\left(x_{0}+x_{1} b\right)\left(x_{0}+x_{1} b\right)^{*}=\left(x_{0}+x_{1} b\right)\left(x_{0}^{*}+b^{-1} x_{1}^{*}\right) \\
& =\left(x_{0}+x_{1} b\right)\left(x_{0}^{*}+x_{1} b\right)=x_{0} x_{0}^{*}+x_{0} x_{1} b+x_{1} b x_{0}^{*}+x_{1} x_{1}^{*} \\
& =x_{0} x_{0}^{*}+x_{0} x_{1} b+x_{0} x_{1} b+x_{1} x_{1}^{*} \\
& =x_{0} x_{0}^{*}+x_{1} x_{1}^{*} .
\end{aligned}
$$

Agora, se $y=\alpha_{1} a_{1}+\cdots+\alpha_{s} a_{s} \in K A$, com $a_{i} \in A$, e $1 \leq i \leq s$, então

$$
\begin{aligned}
y y^{*} & =\left(\alpha_{1} a_{1}+\cdots+\alpha_{s} a_{s}\right)\left(\alpha_{1} a_{1}^{-1}+\cdots+\alpha_{s} a_{s}^{-1}\right) \\
& =\left(\alpha_{1}^{2}+\cdots+\alpha_{s}^{2}\right)+\sum_{\substack{i, j \\
i<j}} \alpha_{i} \alpha_{j}\left(a_{i} a_{j}^{-1}+a_{j} a_{i}^{-1}\right) .
\end{aligned}
$$

Se $a_{i} a_{j}^{-1}$ é um elemento de ordem 2 , então $a_{i} a_{j}^{-1}=a_{j} a_{i}^{-1}$ e, portanto, o suporte do elemento $y y^{*}$ não contém elemento de ordem 2 . E disto temos que

$$
y y^{*}=(\varepsilon(y))^{2}+\sum_{a \in L} \alpha_{a}\left(a+a^{-1}\right) .
$$

Como uma conseqüência, temos que para todo $x \in V(K G)$ vale

$$
x x^{*}=1+\sum_{a \in L} \alpha_{a}\left(a+a^{-1}\right)
$$

Teorema 4.1.3 Sejam K um corpo finito de caracteristica 2 e G um 2-grupo finito contendo um subgrupo abeliano $A$ de indice 2 e um elemento $b$ de ordem 2 que inverte cada 
elemento de A. Então

$$
\left|V_{*}(K G)\right|=|K|^{\frac{3|A|+|A[2]|-2}{2}} .
$$

Prova: Considerando $L$ um sistema completo de representantes do subconjunto $\left\{a^{-1}, a \mid a \in\right.$ $A \backslash A[2]\}$, vemos que o número de elementos de $L$ é $\ell=\frac{|A|-|A[2]|}{2}$. Logo, pelo Lema 4.1.2, a ordem do subgrupo $S_{K}(G)$ é no máximo $|K|^{\ell}$. Provaremos que esta é exatamente a ordem de $S_{K}(G)$. Para isto, basta-nos mostrar que para qualquer $z$ da forma

$$
z=1+\sum_{a \in L} \alpha_{a}\left(a+a^{-1}\right) \quad\left(\alpha_{a} \in K\right)
$$

existe $x \in V(K G)$ tal que $x x^{*}=z$.

Se $z=1+\alpha_{1}\left(a_{1}+a_{1}^{-1}\right)+\alpha_{2}\left(a_{2}+a_{2}^{-1}\right)+\cdots+\alpha_{s}\left(a_{s}+a_{s}^{-1}\right)$, então colocamos $x_{0}=$ $\alpha_{1} a_{1}+\alpha_{2} a_{2}+\cdots+\alpha_{s} a_{s}$ e $x_{1}=1+x_{0}$. Então temos que

$$
\varepsilon\left(x_{0}\right)+\varepsilon\left(x_{1}\right)=1+2\left(\alpha_{1}+\alpha_{2}+\cdots+\alpha_{s}\right)=1
$$

e

$$
x=x_{0}+x_{1} b \in V(K G) .
$$

Além do mais, vemos que

$$
\begin{aligned}
x x^{*} & =x_{0} x_{0}^{*}+x_{1} x_{1}^{*}=x_{0} x_{0}^{*}+\left(1+x_{0}\right)\left(1+x_{0}^{*}\right) \\
& =x_{0} x_{0}^{*}+1+x_{0}^{*}+x_{0}+x_{0} x_{0}^{*} \\
& =1+x_{0}+x_{0}^{*} .
\end{aligned}
$$


Isto é,

$$
\begin{aligned}
x x^{*} & =1+\left(\alpha_{1} a_{1}+\cdots+\alpha_{s} a_{s}\right)+\left(\alpha_{1} a_{1}+\cdots+\alpha_{s} a_{s}\right)^{*} \\
& =1+\left(\alpha_{1} a_{1}+\cdots+\alpha_{s} a_{s}\right)+\left(\alpha_{1} a_{1}^{-1}+\cdots+\alpha_{s} a_{s}^{-1}\right) \\
& =1+\alpha_{1}\left(a_{1}+a_{1}^{-1}\right)+\cdots+\alpha_{s}\left(a_{s}+a_{s}^{-1}\right)=z .
\end{aligned}
$$

Deste modo, temos que a ordem do subgrupo $S_{K}(G)$ é igual a $|K|^{\ell}$. Visto que $|V(K G)|=|K|^{|G|-1}=|K|^{2|A|-1}$, pelo Lema 4.1.1, obtemos que

$$
\begin{aligned}
\left|V_{*}(K G)\right| & =|K|^{2|A|-1} \cdot|K|^{-\frac{|A|-|A[2]|}{2}} \\
& =|K|^{\frac{3|A|+|A[2]|-2}{2}} .
\end{aligned}
$$

Corolário 4.1.4 Sejam $K$ um corpo finito de característica $2 e$

$$
D_{2^{n+1}}=\left\langle a, b \mid a^{2^{n}}=b^{2}=1, a^{b}=a^{-1}\right\rangle
$$

um grupo diedral de ordem $2^{n+1}$. Então temos que

$$
\left|V_{*}\left(K D_{2^{n+1}}\right)\right|=|K|^{3 \cdot 2^{n-1}} .
$$

Prova: Temos que $D_{2^{n+1}}=A \rtimes\langle b\rangle$, onde $A=\left\langle a \mid a^{2^{n}}=1\right\rangle$. Daí, temos que $|A[2]|=2$ e $|A|=2^{n}$. Portanto, segue que

$$
\left|V_{*}\left(K D_{2^{n+1}}\right)\right|=|K|^{\frac{3.2^{n}+2-2}{2}}=|K|^{3.2^{n-1}} .
$$




\subsection{Quatérnios generalizados}

$\mathrm{O}$ resultado seguinte trata-se de uma mera generalização do Teorema 1.3.1. Aqui utilizamos o mesmo método lá desenvolvido com pequenas modificações. No que se segue, denotamos $A[2]=\left\{a \in A \mid a^{2}=1\right\}$ para $A$ um grupo abeliano.

Teorema 4.2.1 Sejam $K$ um corpo finito de característica 2 e $G$ um 2-grupo finito contendo um subgrupo abeliano $A$ de índice 2 e um elemento $b$ de ordem 4 que inverte cada elemento de $A$. Então o subgrupo unitário $V_{*}(K G)$ é o produto semidireto de $G$ e um subgrupo normal $H$. O subgrupo $H$ é o produto semidireto do 2-grupo abeliano elementar

$$
R=\left\{1+\left(1+b^{2}\right) z b \mid z \in K A\right\}
$$

e o subgrupo abeliano $U$, onde $V_{*}(K A)=A \times U$.

Prova: Seja $G=A \rtimes\langle b\rangle$, onde $A$ é um 2-grupo abeliano de índice 2 e $b$ de ordem 4 com $a^{b}=a^{-1}$, para todo $a \in A$.

Sendo $A$ um 2-grupo abeliano finito, pelo Teorema 1.3.2, temos que $V_{*}(K A)=A \times U$ $\mathrm{e}$

$$
\left|V_{*}(K A)\right|=\left|A^{2}[2]\right| \cdot|K|^{\frac{1}{2}(|A|+|A[2]|)-1} .
$$

Sendo $G=\langle A, b\rangle$, então cada elemento $x$ em $K G$ pode ser escrito como

$$
x=x_{1}+x_{2} b
$$


$\operatorname{com} x_{i} \in K A, i=1,2$.

Em particular, se $x \in V_{*}(K G)$, então $x=x_{1}+x_{2} b, \operatorname{com} x_{i} \in K A, i=1,2, \varepsilon(x)=$ $\varepsilon\left(x_{1}\right)+\varepsilon\left(x_{2}\right)=1$ e $x x^{*}=1$.

Agora, visto que $G \leq V_{*}(K G)$, se $x \in V_{*}(K G)$, então $x b=x_{1} b+x_{3} \in V_{*}(K G)$, com $x_{3}=x_{2} b^{2} \in K A$. Portanto, sem perda de generalidades, podemos assumir que $\varepsilon\left(x_{1}\right)=1$ e $\varepsilon\left(x_{2}\right)=0$. Logo, $x_{1} \in V(K A)$ e disto $x$ pode ser escrito como

$$
x=x_{1}\left(1+x_{1}^{-1} x_{2} b\right)
$$

Logo, todo $x \in V_{*}(K G)$ pode ser tomado como $x=x_{1}\left(1+x_{2} b\right)$ com $x_{i} \in K A, i=1,2$, $\varepsilon\left(x_{1}\right)=1$ e $\varepsilon\left(x_{2}\right)=0$. Então temos que

$$
x^{*}=\left(x_{1}\left(1+x_{2} b\right)\right)^{*}=\left(1+x_{2} b\right)^{*} x_{1}^{*}=\left(1+b^{-1} x_{2}^{*}\right) x_{1}^{*} .
$$

Deste modo segue que

$$
\begin{aligned}
x x^{*} & =x_{1}\left(1+x_{2} b\right)\left(1+b^{-1} x_{2}^{*}\right) x_{1}^{*} \\
& =\left(x_{1}+x_{1} x_{2} b\right)\left(x_{1}^{*}+b^{-1} x_{2}^{*} x_{1}^{*}\right) \\
& =x_{1} x_{1}^{*}+x_{1} b^{-1} x_{2}^{*} x_{1}^{*}+x_{1} x_{2} b x_{1}^{*}+x_{1} x_{2} x_{2}^{*} x_{1}^{*} \\
& =x_{1} x_{1}^{*}+x_{1} x_{1}^{*} x_{2} x_{2}^{*}+x_{1} x_{2} b^{-1} x_{1}^{*}+x_{1} x_{2} x_{1} b \\
& =x_{1} x_{1}^{*}\left(1+x_{2} x_{2}^{*}\right)+x_{1} x_{2} x_{1} b^{-1}+x_{1} x_{2} x_{1} b \\
& =x_{1} x_{1}^{*}\left(1+x_{2} x_{2}^{*}\right)+x_{1}^{2} x_{2}\left(b^{-1}+b\right),
\end{aligned}
$$

onde usamos o fato que $a^{b}=a^{-1}$, para todo $a \in A$, implica que $x_{i}^{b}=x_{i}^{*}, i=1,2$. 
Assim, temos que $x \in V_{*}(K G)$ se e somente se

$$
\left\{\begin{array}{l}
x_{1} x_{1}^{*}\left(1+x_{2} x_{2}^{*}\right)=1 \\
x_{1}^{2} x_{2}\left(b^{-1}+b\right)=0
\end{array}\right.
$$

o que equivale a

$$
\left\{\begin{array}{l}
x_{1} x_{1}^{*}\left(1+x_{2} x_{2}^{*}\right)=1 \\
x_{2}\left(1+b^{2}\right)=0
\end{array}\right.
$$

Mas o anulador de $\left(1+b^{2}\right)$ em $K A$ é exatamente

$$
A n n_{K A}\left(1+b^{2}\right)=\left\{\left(1+b^{2}\right) z \mid z \in K A\right\},
$$

e então $x_{2}$ é da forma

$$
x_{2}=\left(1+b^{2}\right) z \quad(z \in K A)
$$

e disto segue que

$$
x_{2} x_{2}^{*}=\left(1+b^{2}\right) z z^{*}\left(1+b^{2}\right)=0 .
$$

Assim, de (1) temos que $x_{1} x_{1}^{*}=1$ e então $x_{1} \in V_{*}(K A)$.

Considere agora o seguinte subgrupo

$$
R=\left\{1+\left(1+b^{2}\right) z b \mid z \in K A\right\}=\prod_{u \in T}\left\langle 1+\lambda u\left(1+b^{2}\right) b \mid \lambda \in K\right\rangle
$$

onde $T$ é um transversal para $\left\langle b^{2}\right\rangle$ em $A$. Temos assim que para todo $z \in K A$,

$$
\begin{aligned}
\left(1+\left(1+b^{2}\right) z b\right)\left(1+\left(1+b^{2}\right) z b\right)^{*} & =\left(1+\left(1+b^{2}\right) z b\right)\left(1+b^{-1} z^{*}\left(1+b^{2}\right)\right) \\
& =\left(1+\left(1+b^{2}\right) z b\right)\left(1+\left(1+b^{2}\right) b^{-1} z^{*}\right) \\
& =\left(1+\left(1+b^{2}\right) z b\right)\left(1+\left(1+b^{2}\right) b z^{*}\right) \\
& =\left(1+\left(1+b^{2}\right) z b\right)\left(1+\left(1+b^{2}\right) z b\right) \\
& =1+\left(1+b^{2}\right) z b+\left(1+b^{2}\right) z b \\
& =1
\end{aligned}
$$


pois $\left(1+b^{2}\right) b^{-1}=\left(1+b^{2}\right) b^{3}=\left(1+b^{2}\right) b$ e $z^{b}=z^{*}$, para todo $z \in K A$.

Assim, $R$ é um 2-grupo abeliano elementar de ordem $|K|^{\frac{1}{2}|A|}$ e $R \subseteq V_{*}(K G)$. Observamos, por (2), que $1+x_{2} b \in R$. Logo, vemos que $V_{*}(K G)$ é gerado pelos grupos $G$, $V_{*}(K A)$ e $R$.

Além disso, para todo $z \in K A$, nós temos que

$$
b^{-1}\left(1+\left(1+b^{2}\right) z b\right) b=1+\left(1+b^{2}\right) b^{-1} z b^{2}=1+\left(1+b^{2}\right) z^{*} b \in R
$$

e para cada $\omega \in V_{*}(K A)$, temos

$$
\begin{aligned}
\omega^{-1}\left(1+\left(1+b^{2}\right) z b\right) \omega & =1+\left(1+b^{2}\right) \omega^{-1} z b \omega \\
& =1+\left(1+b^{2}\right) \omega^{-1} \omega^{*} z b \\
& =1+\left(1+b^{2}\right)\left(\omega^{*}\right)^{2} z b \in R
\end{aligned}
$$

porque

$$
b \omega=\omega^{*} b=\omega^{-1} b .
$$

Deste modo, $R$ é um subgrupo normal em $V_{*}(K G)$ e $R \cap U=\langle 1\rangle$, onde $V_{*}(K A)=A \times U$.

Considere $H=\langle R, U\rangle$. Como visto acima, temos que $\omega^{-1} R \omega \subseteq R$, para todo $\omega \in$ $V_{*}(K A)$, e disto segue que $H$ é um produto semidireto do subgrupo normal $R$ e o subgrupo abeliano $U$, i.e, $H=U \ltimes R$ 
Portanto, temos que $V_{*}(K G)=\langle G, H\rangle$. Das identidades acima, vemos que $H$ é um subgrupo normal de $V_{*}(K G)$ e que

$$
V_{*}(K G)=G \ltimes H=G \ltimes(U \ltimes R) .
$$

Corolário 4.2.2 Sejam $K$ um corpo finito de característica 2 e $G$ um 2-grupo finito contendo um subgrupo abeliano $A$ de indice 2 e um elemento $b$ de ordem 4 tal que $a^{b}=a^{-1}$ para todo $a \in A$. Então temos que

$$
\left|V_{*}(K G)\right|=2 .\left|A^{2}[2]\right||K|^{|A|+\frac{1}{2}|A[2]|-1} .
$$

Prova: Pelo Teorema 4.2.1, temos que

$$
V_{*}(K G)=G \ltimes(U \ltimes R), \quad \operatorname{com}|R|=|K|^{\frac{1}{2}|A|} .
$$

Sabemos também que $V_{*}(K A)=A \times U$ tem ordem

$$
\left|V_{*}(K A)\right|=\left|A^{2}[2]\right||K|^{\frac{1}{2}(|A|+|A[2]|)-1} .
$$

Logo, $|U|=|A|^{-1} \cdot\left|A^{2}[2]\right| .|K|^{\frac{1}{2}(|A|+|A[2]|)-1}$.

Portanto, segue que

$$
\begin{aligned}
\left|V_{*}(K G)\right| & =|G| \cdot|U| \cdot|R| \\
& =2 \cdot|A| \cdot|A|^{-1} \cdot\left|A^{2}[2]\right||K|^{\frac{1}{2}(|A|+|A[2]|)-1} \cdot|K|^{\frac{1}{2}|A|} \\
& =2 \cdot\left|A^{2}[2]\right| \cdot|K|^{|A|+\frac{1}{2}|A[2]|-1} .
\end{aligned}
$$


Corolário 4.2.3 Sejam $K$ um corpo finito de caracteristica 2 e

$$
Q_{2^{n+1}}=\left\langle a, b \mid a^{2^{n}}=1, b^{2}=a^{2^{n-1}}, a^{b}=a^{-1}\right\rangle
$$

um grupo quatérnio de ordem $2^{n+1}$. Então temos que

$$
\left|V_{*}\left(K Q_{2^{n+1}}\right)\right|=4 \cdot|K|^{2^{n}}
$$

Prova: Temos que $Q_{2^{n+1}}=A \rtimes\left\langle b \mid b^{4}=1\right\rangle$ com $A=\left\langle a \mid a^{2^{n}}=1\right\rangle$. Logo, temos que $|A|=2^{n},|A[2]|=2$ e $\left|A^{2}[2]\right|=2$. Portanto, concluímos pelo Corolário 4.2 .2 que

$$
\left|V_{*}\left(K Q_{2^{n+1}}\right)\right|=4|K|^{2^{n}}
$$




\section{Referências}

[1] Bovdi, A.A., The group of units of a group algebra of characteristic p, Publ. Math. Debrecen 52 (1-2) (1998), 193-244.

[2] Bovdi, A.A., Erdei, L., Unitary units in modular group algebras of groups of order 16, Technical Report Universitas Debrecen, Dept. of Math., L. Kossuth Univ. 96/4 (1996), 1-16.

[3] Bovdi, A.A., Gudivok, P.M., Semirot, M.S., Normal group rings. Ukrain Mat. Zh., 37, 3-8 (1985) (Russian).

[4] Bovdi, A.A., Szakács, A., Unitary subgroup of the group of units of a modular group algebra of a finite abelian p-group, Mat. Zametki 45, n.6 (1989), 23-29.

[5] Bovdi, A.A., Szakács, A., A basis for the unitary subgroup of the group of units in a finite commutative group algebra, Publ. Math. Debrecen 46 (1-2) (1995), 97-120.

[6] Bovdi, V., Kovács, L.G., Unitary units in modular group algebras,Manuscripta Math. 84 (1994), 57-72.

[7] Bovdi, V., Kovács, L.G., Sehgal, S.K., Symmetric units in modular group algebra, Comm. Algebra 24, n.3 (1996), 803-808.

[8] Bovdi, V., Rozgonyi, T., On the unitary subgroup of modular group algebras, Acta Acad. Paedagogicae Nyíregyháza 13/ (1992), 13-17. 
[9] Bovdi, V. e Rosa, A.L., On the order of the unitary subgroup of a modular group algebra,Comm. Algebra,28 (4) (2000), 1897-1905.

[10] Chuang, C.L., Lee, P.H., Unitary elements in simple artinian rings, J. Algebra 176, 449-459 (1995).

[11] Robinson, D.J.S., A course in the theory of groups, Springer-Verlag, Berlin-Heidelberg-New York, 1996.

[12] Suzuki, M., Group theory I, Springer-Verlag, Berlin-Heidelberg-New York, 1982. 